\title{
ESPECTROS DE ENERGÍA DE MOVIMIENTOS FUERTES REGISTRADOS EN MÉXICO
}

\author{
Benjamín Huerta Garnica ${ }^{(1)}$ y Eduardo Reinoso Angulo $^{(1)(2)}$
}

\begin{abstract}
RESUMEN
Se hace un análisis de la energía obtenida a partir de acelerogramas de algunos sismos fuertes obtenidos en la zona epicentral y en el valle de México. Primero se comparan los espectros de energía de entrada de algunos registros cercanos al epicentro con los espectros de respuesta de aceleración y de velocidad y se correlacionan cualitativamente con el daño. Se analiza la ecuación de energía, las definiciones de energía absoluta y relativa y la variación de éstas en el tiempo. Para sitios instrumentados en zona de lago del valle de México se comentan brevemente las características de atenuación y la variación de energía con la magnitud y dirección del movimiento. Además, se comenta la variación de los espectros de energía de entrada y de histéresis con la resistencia y con la demanda de ductilidad. Por último, se presentan los espectros elásticos de energía de entrada y de energía histerética normalizada para el sismo del 25 de abril de 1989 y los correspondientes mapas con curvas de igual energía para cuatro diferentes periodos estructurales. También se presentan curvas de igual energía y periodo estructural de dos segundos para cuatro sismos que tuvieron una intensidad importante en el valle de México.
\end{abstract}

\section{SUMMARY}

An analysis of the input energy in the valley of Mexico for some strong earthquakes is presented. Input-energy, acceleration and velocity response spectra of epicentral stations are qualitatively correlated with the damage. Some results were included to discuss the definitions of absolute and relative energy and the time variation of the energy. The attenuation characteristics and the energy variation with magnitude and direction of motion for places at the lakebed zone of the valley of Mexico are briefly commented. Besides, the variation of the input energy spectra and the hysteresis spectra with respect to the strength and ductility demand is commented. Finally, the elastic spectra of input energy and normalized hysteretic energy for the earthquake of April 251989 and the corresponding maps with curves of equal energy for four structural periods are shown. These curves are drawn for a structural period of two seconds for four earthquakes that had an important intensity in the valley of Mexico.

Artículo recibido el 15 de diciembre de 2000 y aprobado para su publicación el 26 de septiembre de 2001. Se aceptarán comentarios y/o discusiones hasta cinco meses después de su publicación.

(1) ERN Ingenieros Consultores, Desierto de los Leones No. 46 Colonia San Ángel, 01000 México, DF. bhg@ern.com.mx

(2) Instituto de Ingeniería, UNAM, Ciudad Universitaria, 04510 México, DF. ere@pumas.iingen.unam.mx 


\section{INTRODUCCIÓN}

Los terremotos son fenómenos en que enormes cantidades de energía mecánica acumulada en las zonas de interacción entre placas tectónicas durante décadas o incluso siglos son liberadas en periodos muy cortos de tiempo. Gracias a las aportaciones de la ciencia moderna hoy sabemos en qué consiste un terremoto y cuáles son los mecanismos que lo producen. No obstante, la ingeniería sísmica sigue utilizando las fuerzas y desplazamientos en la estructura como parámetros de diseño y reconoce que éstos son sólo una manifestación del evento sísmico y no representan al fenómeno en su totalidad al no tomar en cuenta la historia completa del movimiento. El caso de la ciudad de México es particularmente importante por la gran duración de los sismos.

Los reglamentos de construcción en zonas sísmicas indican que las construcciones deben ser capaces de resistir determinadas fuerzas sísmicas y no exceder las condiciones de servicio para las que están destinadas. La mayoría de las estructuras se diseñan para que durante temblores moderados y severos sus materiales incursionen en comportamiento inelástico para disipar parte de la energía introducida por el sismo. Este comportamiento inelástico provoca daño a los elementos estructurales el cual debe ser tomado en cuenta en el análisis y diseño de la estructura. Recientemente se ha utilizado la distorsión de entrepiso o el máximo desplazamiento relativo entre niveles consecutivos de la estructura para determinar el daño que ésta sufrirá.

Los reglamentos actuales están elaborados para garantizar que las construcciones puedan resistir sismos severos que ocurren en un determinado periodo de retorno sin que colapsen. Sin embargo, no garantizan que las construcciones presenten un buen comportamiento sísmico ante diferentes niveles de movimiento del terreno, es decir, que los daños puedan controlarse. Esto ha dado lugar a intensas discusiones sobre la necesidad de revisar estos códigos y modificar la metodología de diseño empleada. Así han aparecido diversos métodos de diseño basados en desempeño que consideran el control de daño. Los conceptos de energía que se expondrán posteriormente pueden ser aplicados a estos métodos y a los existentes. Una de las condiciones que establecen los métodos de diseño basados en conceptos de energía es que la capacidad de admitir y disipar energía de la estructura debe ser mayor que la demanda de energía que puede producir el sismo de diseño, considerando distintos niveles de movimiento del terreno. Sin embargo, esto no es suficiente y debe complementarse con controles de daño tales como la demanda de desplazamientos y de ductilidades garantizando también que las fuerzas y momentos actuantes sean menores que los resistentes.

La tendencia para crear un método de diseño basado en energía involucra, como los otros métodos, la consideración de dos aspectos: el primero se relaciona al establecimiento de los niveles de peligro sísmico en función de la energía correspondiente y el segundo con la evaluación de la absorción de energía y la capacidad de disipación de energía de la estructura. El objetivo de este trabajo es introducir una propuesta que podría contribuir a la solución del primero de los aspectos antes mencionados. 


\section{MOVIMIENTO FUERTE DEL TERRENO EN LA ZONA EPICENTRAL}

\section{Espectros de aceleración y de velocidad}

Los espectros de diseño que contienen los reglamentos generalmente se obtienen trazando una envolvente de varios espectros de respuesta de un oscilador de un grado de libertad. Los espectros de respuesta representan los valores de respuesta máxima de varios osciladores caracterizados por su periodo natural de vibración y por un amortiguamiento dado, usualmente 5 por ciento. Por definición, estos espectros no consideran la contribución del movimiento del acelerograma completo sino solamente de los valores máximos. Se pueden obtener espectros de respuesta muy parecidos para dos acelerogramas con diferente forma, duración y contenido de frecuencias pero que produjeron la misma respuesta máxima del oscilador. Sin embargo, estos acelerogramas pueden introducir energía a la estructura con diferentes características e intensidades.

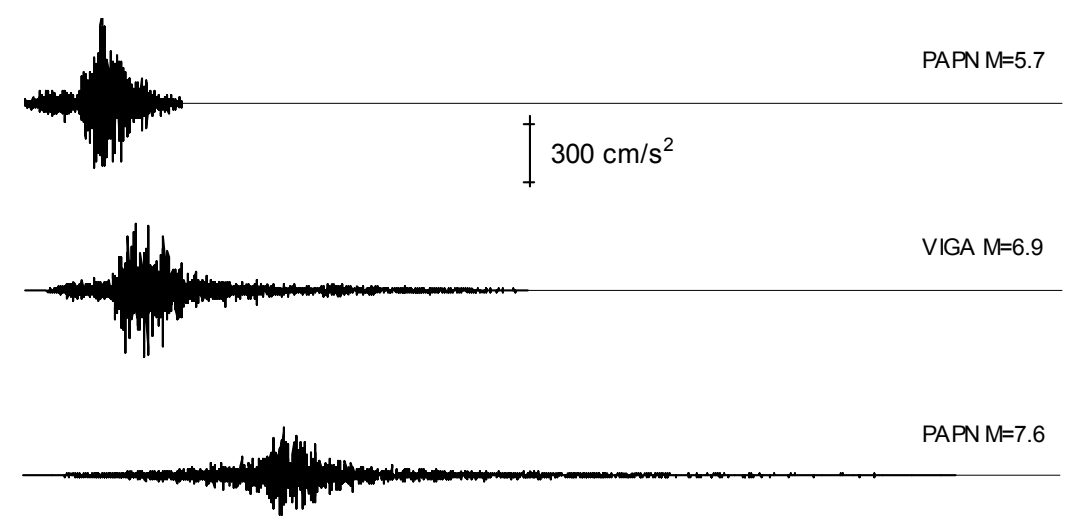

VILE M=8.1

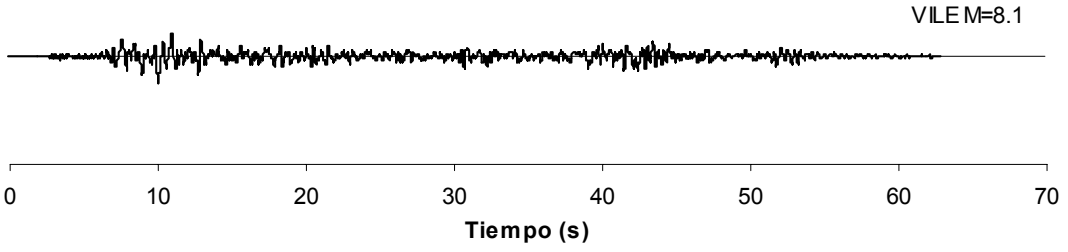

Figura 1. Registros de aceleración del terreno para cuatro sismos de diferentes magnitudes. Los registros se encuentran sobre roca a la misma distancia de la superficie de ruptura $(19 \mathrm{~km})$

Recientemente se ha reconocido que las ordenadas de un espectro de respuesta no están siempre correlacionadas con el daño en los distintos sistemas estructurales. Como ejemplo se muestran en la fig. 1 los registros obtenidos en estaciones sobre roca localizadas a la misma distancia de la superficie de ruptura (alrededor de $19 \mathrm{~km}$ ) durante cuatro sismos de diferentes magnitudes $(5.7,6.9,7.6$ y 8.1 correspondientes a los sismos del 8 de febrero de 1988, 25 de abril de 1989, 21 y 19 de septiembre de 1985, respectivamente) originados en la zona de subducción de las costas del Océano Pacífico mexicano. Debido a que estos registros están todos sobre roca y que tienen la misma distancia a la superficie de ruptura, es de esperarse que sus diferencias sean atribuidas solo a la magnitud ya que la secuencia de ruptura es un efecto que generalmente se 
desprecia con fines de predicción de movimiento fuerte. Cabe destacar que de estos sismos el único en el que se registraron daños importantes en las construcciones de su zona epicentral fue durante el sismo del 19 de septiembre de 1985, por lo que se puede pensar que este registro debería tener mayor amplitud en la aceleración del terreno. Sin embargo, los registros provenientes del sismo de menor magnitud presentan mayor aceleración del terreno que la de los registros de mayor magnitud. Por ejemplo: para el registro PAPN correspondiente al sismo de magnitud 5.7 se tiene una aceleración máxima del terreno de $434 \mathrm{~cm} / \mathrm{s}^{2}$, que es cuatro veces mayor que la aceleración del registro VILE $\left(104 \mathrm{~cm} / \mathrm{s}^{2}\right)$ obtenida de un sismo con magnitud de 8.1. A pesar de que Nau y Hall (1984) mostraron que la aceleración máxima de terreno solo está bien correlacionada con la respuesta de estructuras de periodo corto, durante los sismos mencionados para la fig. 1 sólo existieron daños para el sismo del 19 de septiembre, y no para los otros que tienen mayores aceleraciones del terreno.

Esta observación confirma el hecho de que la aceleración del terreno no está correlacionada con la magnitud del sismo y por lo tanto tampoco está correlacionada con el daño ocasionado por éste. En la fig. 2 se muestra cómo varían los espectros de respuesta de aceleración con la magnitud. Para estas gráficas se utilizaron varios registros con la misma distancia a la superficie de ruptura de ocho sismos con diferentes magnitudes $(5.2,5.3,5.7,5.8,6.8,7.3,7.6 \mathrm{y}$ 8.1). Estas gráficas se calcularon obteniendo la envolvente de los espectros de respuesta de las estaciones seleccionadas en ambas direcciones y suavizando las variaciones de las ordenadas en la magnitud y periodo mediante una regresión matemática. A pesar de este suavizado, la tendencia de cada espectro (no se muestra en este trabajo) se refleja razonablemente bien en la fig. 2. Se observa que en los espectros de aceleración las ordenadas son menores tanto para los sismos pequeños $(M<5.5)$ como para los grandes $(M>7.0)$, mientras que son máximas para sismos de mediana magnitud $(M \approx 6.0)$. Esto no es congruente con el daño que han producido los sismos de gran magnitud en la zona epicentral, por lo que se puede confirmar que los espectros de aceleración, que incluyen la aceleración máxima del terreno (para $\mathrm{T}=0 \mathrm{~s}$ ), no están correlacionados con el daño que pueden sufrir las estructuras.

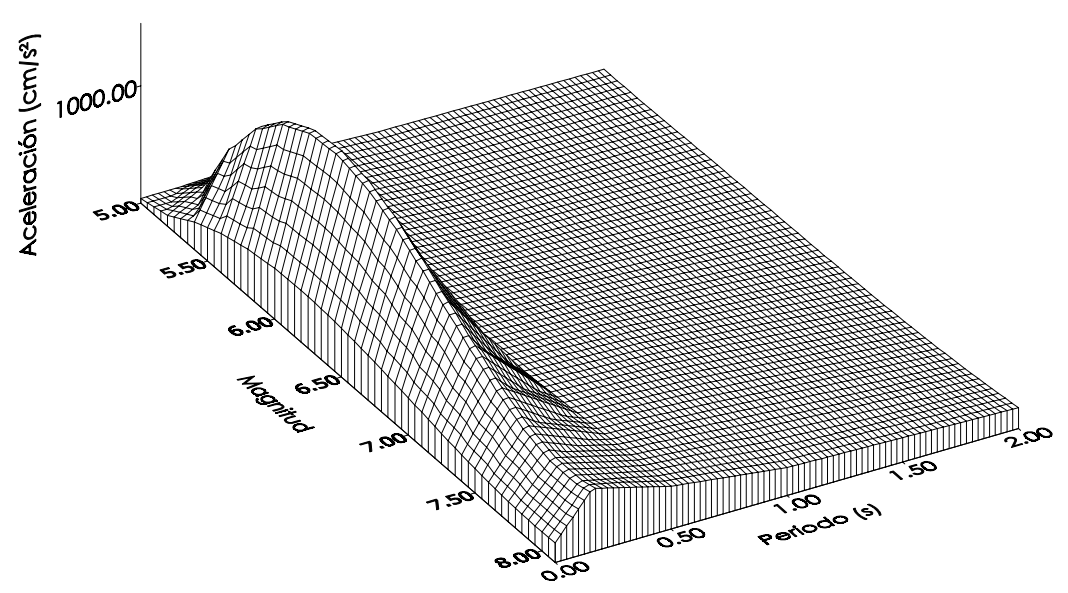

Figura 2. Representación espacial de los espectros de respuesta de aceleración para sismos de subducción con diferente magnitud; los registros corresponden a sitios sobre el área epicentral 
Al comparar los espectros de respuesta de velocidad (fig. 3) obtenidos de la misma forma y para los mismos registros utilizados en los espectros de aceleración, se observa que para periodos cortos $(\mathrm{T}<0.5 \mathrm{~s})$ estas ordenadas espectrales siguen la misma tendencia que las de aceleración; es decir, que las ordenadas disminuyen para sismos de mayor magnitud. Sin embargo, para periodos largos las ordenadas espectrales se incrementan, aunque no de manera importante, con la magnitud. Esto indica que la velocidad espectral está mejor correlacionada con la magnitud del sismo y con el daño provocado por éste. Sin embargo, el daño observado en todas las estructuras, incluyendo las de periodo corto, no puede atribuirse a estas pequeñas diferencias. Housner (1975) reconoció que la velocidad espectral está mejor correlacionada con el daño que la aceleración y varios investigadores han observado que existe una correlación todavía mejor de la velocidad al cuadrado, que es una medida de energía, con el daño.

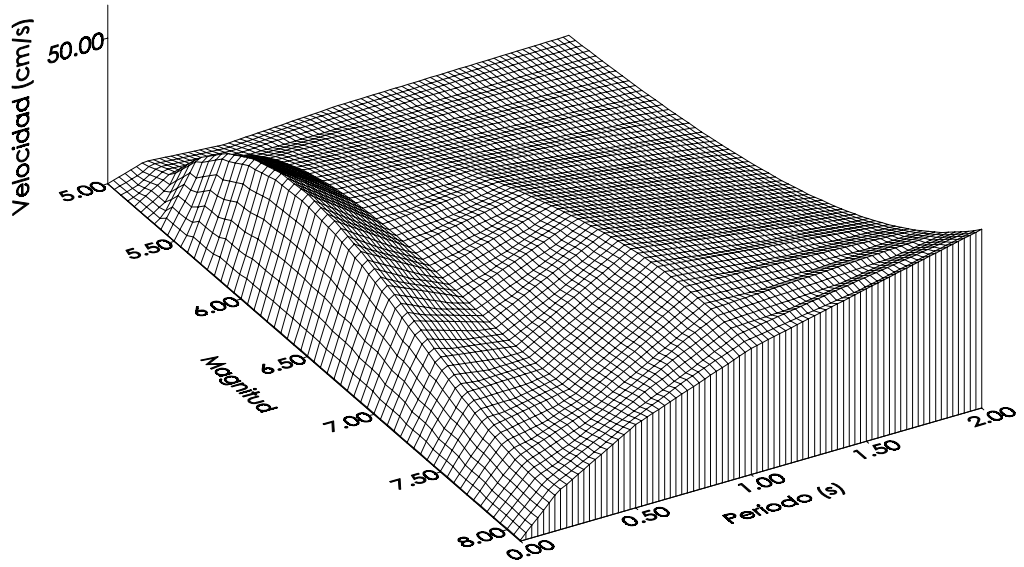

Figura 3. Representación espacial de los espectros de respuesta de velocidad para sismos de subducción con diferente magnitud; los registros corresponden a sitios sobre el área epicentral

Como se aprecia en la fig. 1, los registros obtenidos de sismos severos tienen mayor duración, diferente contenido de frecuencias y, como se mostrará en adelante, mayor energía que los registros provenientes de sismos pequeños y moderados. La duración del movimiento es directamente proporcional a la magnitud del sismo; por lo tanto, esta duración también puede ser un parámetro que se podría utilizar para estimar el daño en estructuras. Una análisis más detallado sobre la duración y sus efectos se encuentra en el trabajo de Reinoso y Ordaz (2001). El hecho de que la energía elástica de entrada sea un resultado que toma en cuenta la amplitud y duración del movimiento ha promovido su uso en análisis de riesgo sísmico.

\section{Espectros de energía}

Como en los espectros de respuesta de aceleración y velocidad, los espectros de respuesta de energía se obtienen graficando la energía máxima de varios osciladores definidos por su periodo y amortiguamiento. Los espectros elásticos de energía de entrada calculados como se indicará más adelante, muestran claramente que existe una relación estrecha de las ordenadas espectrales de energía con la magnitud del sismo y por lo tanto con el daño. Esto se aprecia en la 
representación espacial de la fig. 4 obtenida de la misma forma que las figs. 2 y 3. Se observa un comportamiento muy similar al de los anteriores espectros para periodos cortos $(\mathrm{T}<0.5 \mathrm{~s})$; para periodos mayores la energía permanece baja y constante para magnitudes menores a 7.5 grados pero se incrementa considerablemente cuando el sismo tiene una magnitud mayor o igual a ésta. Para estas gráficas se observa que existe una mejor correlación de la energía de entrada con el daño que los otros espectros ya que los sismos que produjeron daños importantes a las estructuras (y que pueden ocasionarlos en sismos futuros) son los que tienen magnitudes mayores de 7.0 grados. Sin embargo, existen otras formas de energía como la energía histerética normalizada (Terán-Gilmore, 1996) que como se indicará más adelante sí tienen una correlación directa con el daño.

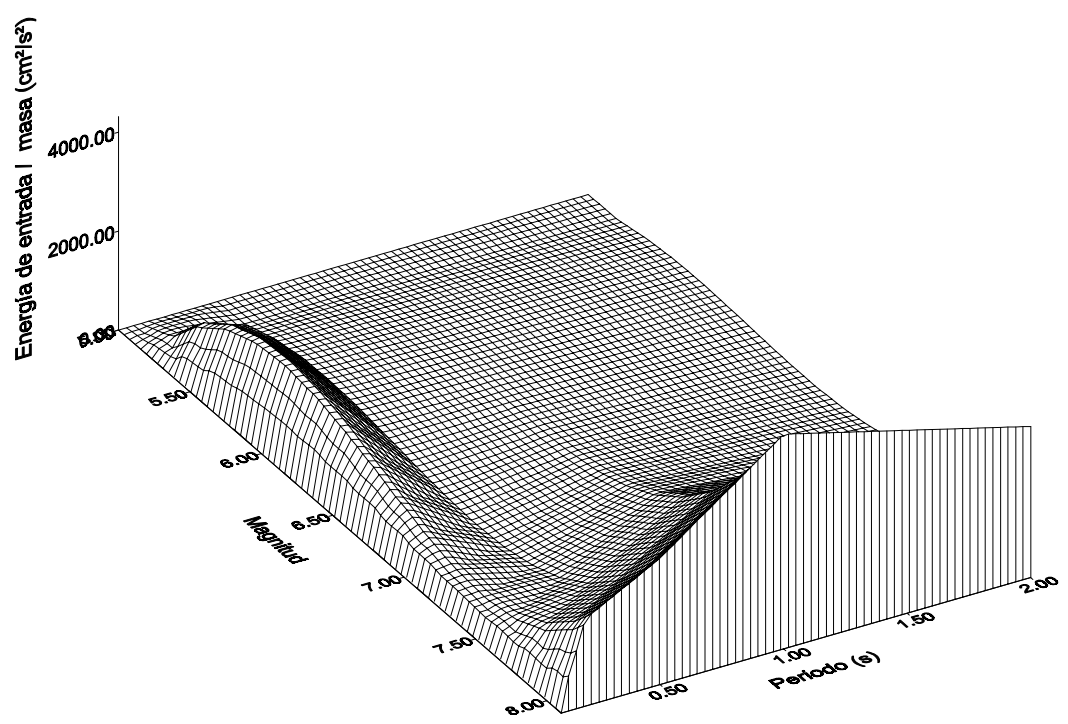

Figura 4. Representación espacial de los espectros de respuesta de energía de entrada para sismos de subducción con diferente magnitud; los registros corresponden a sitios sobre el área epicentral

\section{CONCEPTOS DE ENERGÍA}

Housner (1956) propuso por primera vez una metodología de diseño basada en energía. En años recientes estos conceptos han llamado la atención de numerosos autores (Akiyama, 1985; Uang y Bertero, 1988 y Fajfar et al., 1992). Terán-Gilmore (1996) propuso un método de diseño sísmico basado en desempeño usando conceptos de energía en el cual plantea el siguiente enfoque de demanda-suministro de energía (Uang y Bertero, 1988)

Demanda Sísmica de Energía $\leq$ Suministro Sísmico de Energía

complementado con el uso del índice de daño de Park y Ang. La ec.1 plantea que la entrada o demanda de energía (la energía que el sismo introduce a la estructura) no debe ser mayor que el suministro de energía (mecanismos de almacenamiento y disipación de energía). Por lo tanto, parece razonable estudiar el peligro sísmico desde el punto de vista de demanda de energía y después compararlo con la vulnerabilidad estructural dada por el suministro de la misma. 
Para que el diseño de una estructura resulte adecuado se debe satisfacer la ec. 1 para todos los niveles sísmicos de diseño considerados, además de complementarse con recomendaciones para el control de daño (Terán-Gilmore, 1996).

Para determinar la demanda o energía de entrada se considera un sistema de un oscilador con un grado de libertad con comportamiento inelástico. La ecuación de movimiento es la siguiente:

$$
m x(t)+c \dot{x}(t)+f_{S}(x, \dot{x})=-m x_{g}(t)
$$

donde $x$ representa el desplazamiento relativo de la masa $m, x_{g}$ es el desplazamiento absoluto del terreno, $c$ es el coeficiente de amortiguamiento viscoso de la estructura y $f_{s}$ representa la fuerza de restitución; el punto sobre la literal indica derivada con respecto al tiempo. La energía de entrada relativa es el trabajo efectuado por una fuerza lateral equivalente en un sistema de base fija (fig. 5a). Para un sistema elástico lineal $f s$ es igual a $k x$, donde $k$ es la rigidez del sistema. El término del lado derecho de la ecuación representa la fuerza lateral a la que está sometida la masa, los términos del lado izquierdo de la ecuación representan las fuerzas de inercia, amortiguamiento viscoso y de restitución. Integrando la ecuación anterior con respecto a $x$ para obtener el trabajo que realizan estas fuerzas (la energía), se obtiene lo siguiente (Uang y Bertero, 1988):

$$
\int m x d x+\int c \dot{x} d x+\int f s d x=-\int m x_{g} d x
$$

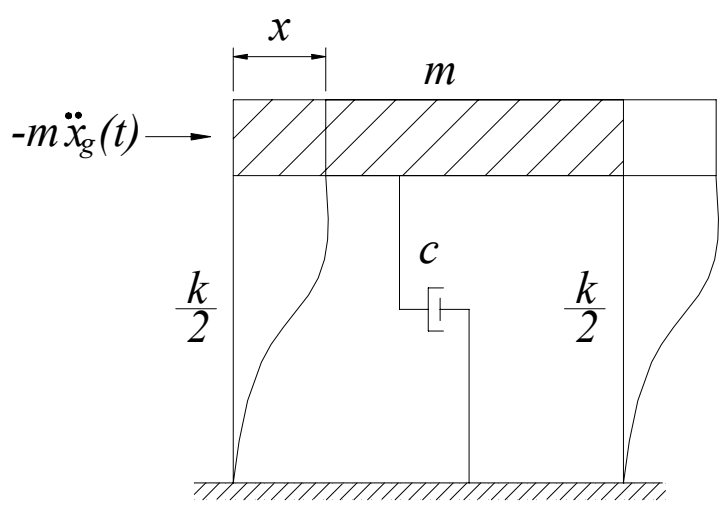

(a)

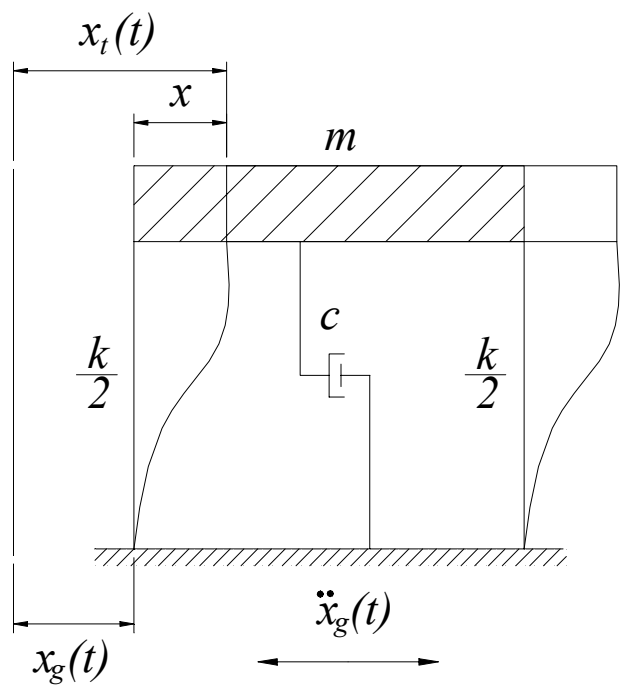

(b)

Figura 5. Modelos de un sistema de 1GDL para las dos definiciones de energía de entrada (Uang y Bertero, 1990): (a) modelo de base fija y (b) modelo de base móvil 
La suma de estas energías debe equilibrar la energía de entrada impuesta a la estructura por el sismo y así la energía de entrada relativa está dada por el término de la derecha de la ecuación anterior:

$E_{I}=-\int m \ddot{x}_{g} d x$

Las contribuciones individuales del lado izquierdo de la ec. 3 representan la energía cinética relativa $\left(E_{C}\right)$, de amortiguamiento viscoso $\left(E_{A}\right)$ y de restitución $\left(E_{D}+E_{H}\right)$. La energía cinética relativa $\left(E_{C}\right)$ es producida por la fuerza de inercia de la masa y esta asociada a la velocidad relativa de la masa, como se muestra a continuación:

$E_{C}=\int m \ddot{x} d x=\int m \dot{x} d \dot{x}=\frac{m \dot{x}^{2}}{2}$

La energía disipada causada por el amortiguamiento viscoso $\left(E_{A}\right)$ se puede expresar como:

$E_{A}=\int c \dot{x} d x=\int c \dot{x}^{2} d t$

Cuando el sistema presenta comportamiento inelástico la energía de restitución se puede descomponer en energía de deformación elástica $\left(E_{D}\right)$ más energía de histéresis $\left(E_{H}\right)$. La energía de deformación elástica $\left(E_{D}\right)$ es la fuerza de la rigidez del sistema que desarrolla energía potencial asociada al desplazamiento:

$E_{D}=\int k x d x=\frac{k x^{2}}{2}$

La energía que es disipada por deformación plástica denominada también energía de histéresis $\left(E_{H}\right)$ se obtiene restando la energía de deformación elástica $\left(E_{D}\right)$ a la energía de restitución:

$E_{H}=\int f_{S}(x, \dot{x}) d x-E_{D}$

Escrita de la siguiente forma, la ec. 3 representa el balance de energía en una estructura:

$E_{I}=E_{C}+E_{A}+E_{D}+E_{H}$

En esta ecuación puede considerarse que el término de la izquierda $\left(E_{I}\right)$ representa la demanda o entrada de energía que el sismo introduce a la estructura y el término de la derecha representa el suministro sísmico de la misma. Esta ecuación se puede representar esquemáticamente por el estanque de agua con dos orificios mostrado en la fig. 6 (Popov et al., 
1993). Para que el tanque opere eficientemente, su capacidad total dada por la suma de su volumen y las salidas de agua, debe ser mayor que la entrada de agua $\left(E_{I}\right)$. Es decir, la capacidad de admitir energía depende del volumen del tanque $\left(E_{C}+E_{D}\right)$ y del tamaño de los orificios por donde escapa el agua $\left(E_{H}\right.$ y $\left.E_{A}\right)$.

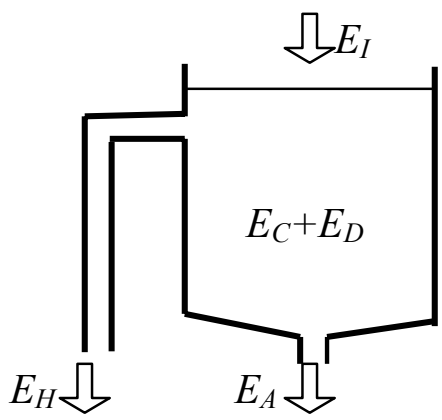

Figura 6. Representación esquemática de la ecuación de balance de energía con ayuda de un tanque de almacenamiento de agua (Popov et al., 1993)

\section{Energía de entrada absoluta}

De igual forma, para un sistema dinámico de base móvil (fig. 5b) se puede definir la energía de entrada absoluta como el trabajo realizado por la fuerza total aplicada en la base de la estructura:

$m \ddot{x}_{t}(t)+c \dot{x}(t)+f s(x, \dot{x})=0$

donde $x_{t}=x_{g}+x$, es el desplazamiento absoluto de la masa. Sustituyendo $x_{t}$ e integrando la ecuación anterior con respecto al desplazamiento relativo de la masa, $x$, se tiene lo siguiente (Uang y Bertero, 1990):

$\int m x_{t}\left(d x_{t}-d x_{g}\right)+\int c \dot{x} d x+\int f_{s} d x=0$

Separando la integral del primer término y ordenando la ecuación se tiene:

$m \dot{x}_{t}^{2} / 2+\int c \dot{x} d x+\int f_{S} d x=\int m \ddot{x}_{t} d x_{g}$

El término de la derecha de la ec. 12 se conoce como energía de entrada absoluta. Se observa que en los términos de la izquierda de ambas definiciones de energía, la energía de amortiguamiento $\left(E_{A}\right)$ y de restitución $\left(E_{D}+E_{H}\right)$ son las mismas y el único término que cambia es el de energía cinética $\left(E_{C}\right)$. 
Ambas definiciones de energía están en función del tiempo, del movimiento del terreno y de la respuesta del oscilador. En la fig. 7 se muestran las historias de energía absoluta y relativa para dos osciladores con periodos $\mathrm{T}=0.3$ y $\mathrm{T}=3.5$ segundos, fracción de amortiguamiento crítico $\xi=5 \%$ y coeficiente sísmico de fluencia $C y=0.15$. Estas historias corresponden al registro de la estación localizada en la Secretaría de Comunicaciones y Transportes componente este-oeste (SCT-EO), del sismo del 19 de septiembre de 1985. En estas gráficas se pueden observar diferencias significativas en las magnitudes de energía $\left(E_{I}\right)$ relativa y absoluta para el periodo de $\mathrm{T}=0.3 \mathrm{~s}$ (figs. $7 \mathrm{a}$ y $7 \mathrm{~b}$ ) y para estructuras con periodo mayor, $\mathrm{T}=3.5 \mathrm{~s}$ (figs. $7 \mathrm{c}$ y $7 \mathrm{~d}$ ). Cuando el periodo de la estructura es significativamente mayor que el periodo predominante del terreno $(\mathrm{T}=3.5 \mathrm{~s}>\mathrm{T}=2 \mathrm{~s})$ la masa de la estructura prácticamente no se mueve, por ello la energía de entrada absoluta es menor que la relativa (Uang y Bertero, 1990). La variación mostrada en la fig. 7 entre ambas definiciones de energía tiene diferencias relativamente pequeñas cuando se comparan las ordenadas máximas de los espectros de energía.

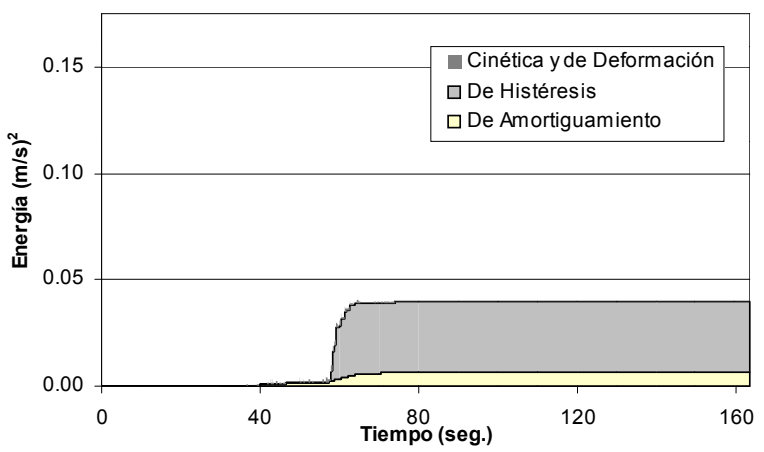

(a) energía relativa $\mathrm{T}=0.3 \mathrm{~s}$

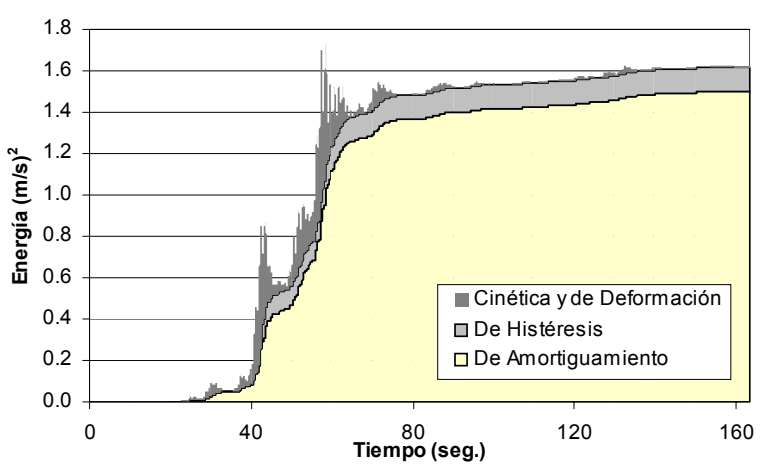

(c) energía relativa $\mathrm{T}=3.5 \mathrm{~s}$

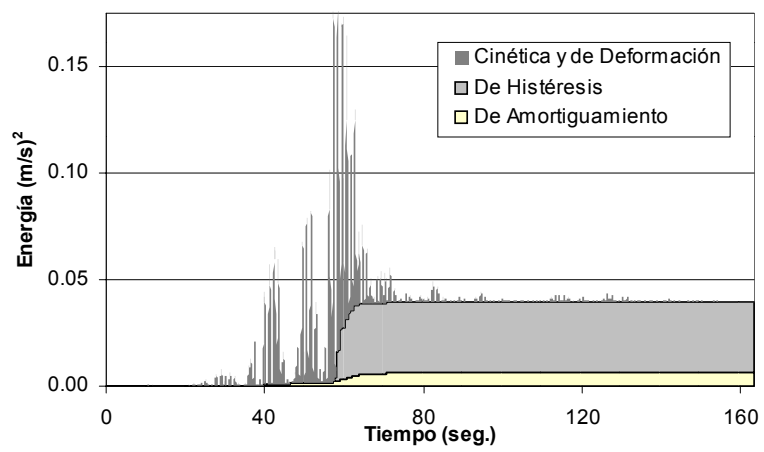

(b) energía absoluta $\mathrm{T}=0.3 \mathrm{~s}$

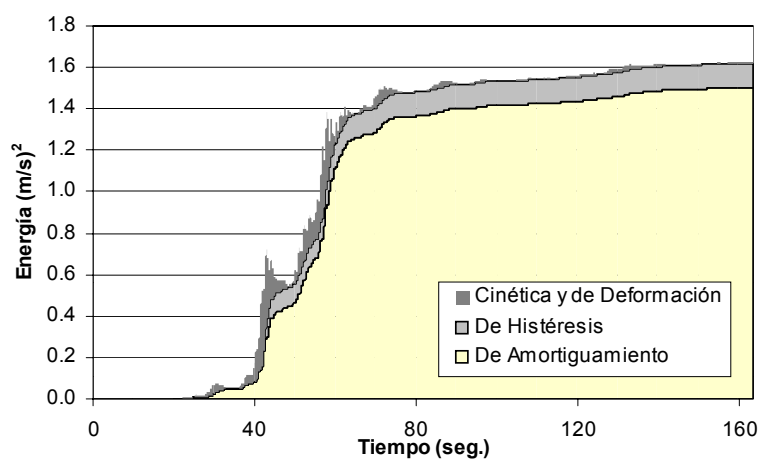

(d) energía absoluta $\mathrm{T}=3.5 \mathrm{~s}$

Figura 7. Variación de las componentes de energía de entrada en el tiempo para el registro SCTEO del sismo del 19 de septiembre de $1985, C y=0.15, \xi=5 \%$

Las diferencias de energía relativa y absoluta son mayores cuando el periodo de la estructura presenta valores extremos, es decir, las diferencias se incrementan para periodos muy largos $(T \rightarrow \infty)$ y para periodos muy cortos $(T \rightarrow 0)$. En un intervalo de periodos cercanos al dominante del terreno la energía relativa y absoluta es idéntica. En la fig. 8 se muestran los 
espectros de energía de entrada relativa y absoluta para el registro de la estación SCT-EO del sismo del 19 de septiembre de 1985 considerando un coeficiente sísmico de fluencia $C y=0.15$. Se observa que los espectros son casi idénticos a excepción de periodos muy cortos $(\mathrm{T}<1)$ en que la energía de entrada absoluta es ligeramente mayor a la relativa y de periodos largos $(\mathrm{T}>4)$ en donde sucede lo contrario. Para muchos de los registros de movimientos registrados en México que fueron analizados se observó poca diferencia de los espectros de energía de entrada relativa y absoluta. Los resultados presentados en este trabajo son para energía relativa, y se puede considerar que los resultados correspondientes a la energía absoluta son similares.

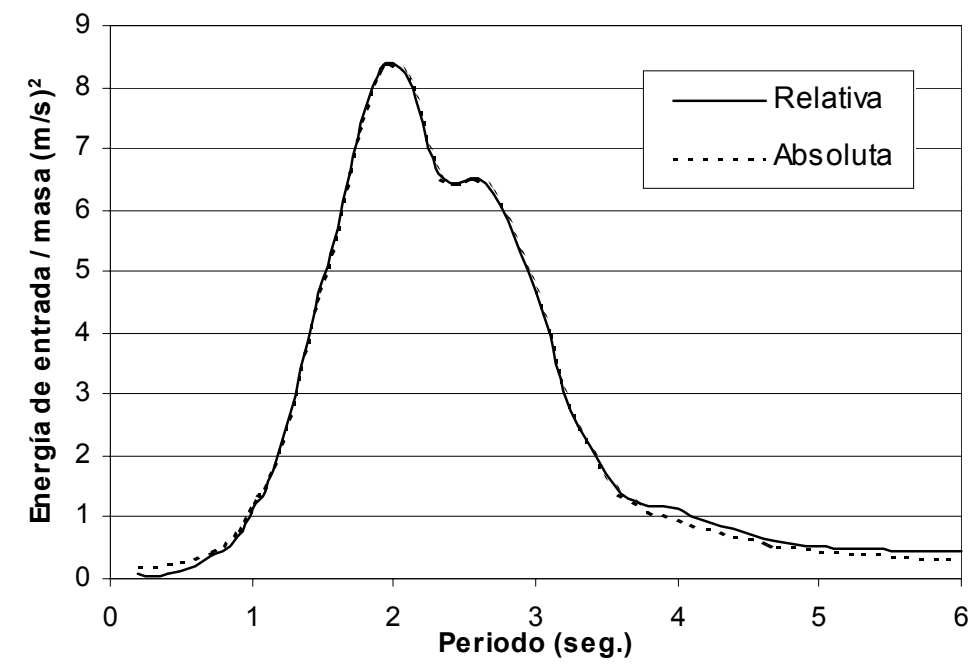

Figura 8. Espectros de energía de entrada relativa y absoluta para el registro SCT-EO del sismo del 19 de septiembre de $1985, C y=0.15, \xi=5 \%$

\section{Energía en estructuras}

Puede decirse que el manejo juicioso de la energía de entrada $\left(E_{I}\right)$ permite representar el daño potencial total del movimiento del terreno provocado por el sismo. No se tiene control ni conocimiento exacto de la cantidad de energía de entrada impuesta a la estructura que se localiza en un sitio determinado ante un sismo futuro; esta varía con la magnitud del sismo, la distancia al epicentro, las características del suelo y características de la estructura. El suministro de energía para una estructura con comportamiento inelástico sujeta a movimientos fuertes del terreno es disipado en parte por deformación inelástica (energía histerética, $E_{H}$ ) y en parte por amortiguamiento viscoso (energía de amortiguamiento, $E_{A}$ ) que en la fig. 6 se representa como el agua que sale del tanque. La energía de histéresis disipada $\left(E_{H}\right)$ es el parámetro de respuesta estructural que está asociada con el daño acumulado de la estructura (Fajfar y Vidic, 1994). Como se verá más adelante, $E_{H}$ normalizada por la máxima energía elástica de deformación está bien correlacionada con el daño estructural.

El diseñador debe analizar si es técnica y económicamente posible equilibrar la energía de entrada sólo con comportamiento elástico de la estructura, es decir, sólo con movimiento, deformación elástica y amortiguamiento de la estructura $\left(E_{C}, E_{D} \mathrm{y} E_{A}\right)$, o qué tanto es conveniente 
disipar parte de la energía de entrada con comportamiento histerético $\left(E_{H}\right)$. La energía de amortiguamiento $\left(E_{A}\right)$ es un parámetro sobre el que el diseñador no tiene mucho control. Para una estructura la fracción de amortiguamiento crítico varía del 2 al 10 por ciento (dependiendo de la fricción interna, fricción en los apoyos, elementos no estructurales y otros mecanismos). La mayoría de las estructuras tiene amortiguamientos del orden de 5 por ciento. En la práctica es más factible aumentar $E_{H}$ a través de comportamiento inelástico en la estructura (deformaciones plásticas) aunque implique daño a los miembros estructurales.

Recientemente se ha reconocido que es posible aumentar $E_{A}$ y $E_{H}$ significativamente y controlar el daño de la estructura a través del uso de dispositivos de disipación de energía. También el diseñador tiene la opción de intentar disminuir $E_{I}$ haciendo uso de las técnicas de aislamiento de base sísmica, no recomendables para suelos blandos como los del Distrito Federal (Bertero, 2000). Para ello es importante conocer los espectros elásticos e inelásticos para $E_{I}$ (Akiyama, 1985; Kuwamura y Galambos, 1989; Fajfar y Vidic, 1994) y otros tipos de energía involucrados en la ecuación de balance de energía.

La energía histerética $\left(E_{H}\right)$ proporciona una idea del número promedio y magnitud promedio de los ciclos en que la estructura incursiona en su rango plástico. El uso exclusivo de la energía histerética $\left(E_{H}\right)$ puede no aportar información suficiente acerca de la importancia que las demandas acumuladas de deformación plástica tienen en el desempeño sísmico de la estructura (Ávila y Terán-Gilmore, 2000). Un parámetro que se correlaciona bien con las demandas acumuladas de deformación plástica en la estructura, y por lo tanto con el daño que esta puede tener, es el de la energía histerética normalizada, $N E_{H}$ (Terán-Gilmore, 1996):

$$
N E_{H}=\frac{E_{H}}{x_{y} F_{y}}
$$

donde $x_{y}$ es el desplazamiento lateral de fluencia y $F_{y}$ es la resistencia de fluencia. El producto $x_{y} F_{y}$ representa una proporción de la energía elástica de deformación almacenada cuando los elementos estructurales son llevados hasta el nivel de fluencia.

\section{ATENUACIÓN DE LA ENERGÍA CON LA DISTANCIA AL ÁREA DE RUPTURA}

Algunos investigadores han estudiado la atenuación de energía mediante modelos utilizados para otros parámetros del movimiento del terreno, observando que la energía es subestimada por las funciones de atenuación en sitios muy cercanos a la falla debido a que estos movimientos provocan generalmente altas demandas de energía a la estructura (Chou y Uang, 2000). El propósito de este trabajo no es el de desarrollar leyes de atenuación de energía sino solamente ilustrar algunas de las características de su comportamiento.

En las figs. 9a y 9b se muestran los espectros elásticos de energía de entrada $\left(E_{I}\right)$ para algunos registros del componente este-oeste de las estaciones de la Red Acelerométrica de Guerrero para el sismo del 19 de septiembre de 1985. La localización de estas estaciones se muestra en el mapa de la fig. 10. En la fig. 9a se observa, para las estaciones que se encuentran 
sobre en el área de ruptura, CALE, VILE y UNIO, la existencia de importantes variaciones tanto en el periodo en que se presenta la máxima ordenada de energía como en la amplitud, lo que dificulta cualquier esfuerzo por predecir estos espectros. Aunque fuera del área de ruptura, la estación AZIH presenta niveles de energía similares a las otras tres estaciones, esto es debido a que está muy cerca de esta área y el efecto de atenuación aún no es visible. Para la estación PAPN, a varias decenas de kilómetros del área de ruptura, se observa claramente una disminución de la energía con la distancia para todos los periodos. Lo mismo sucede para las estaciones cuyos espectros se muestran en la fig $9 \mathrm{~b}$ localizadas lejos del área de ruptura.

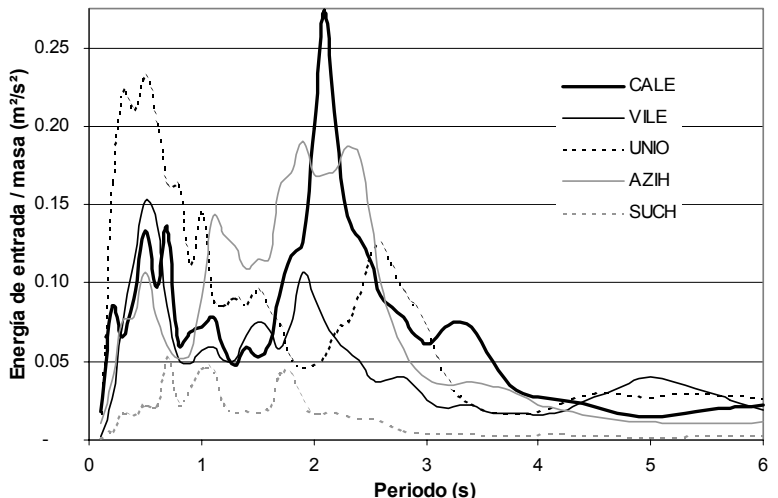

(a) Cercanas al área epicentral

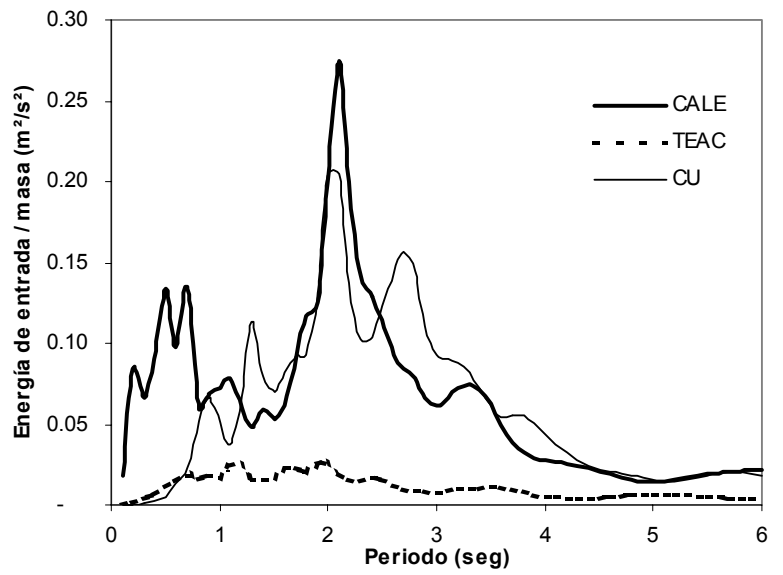

(c) CALE, TEAC y CU (comp. E-O)

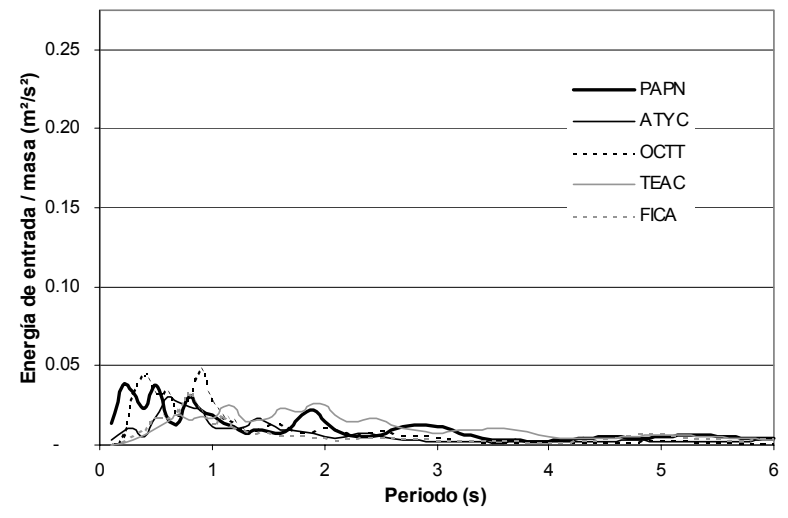

(b) Más alejadas del área epicentral

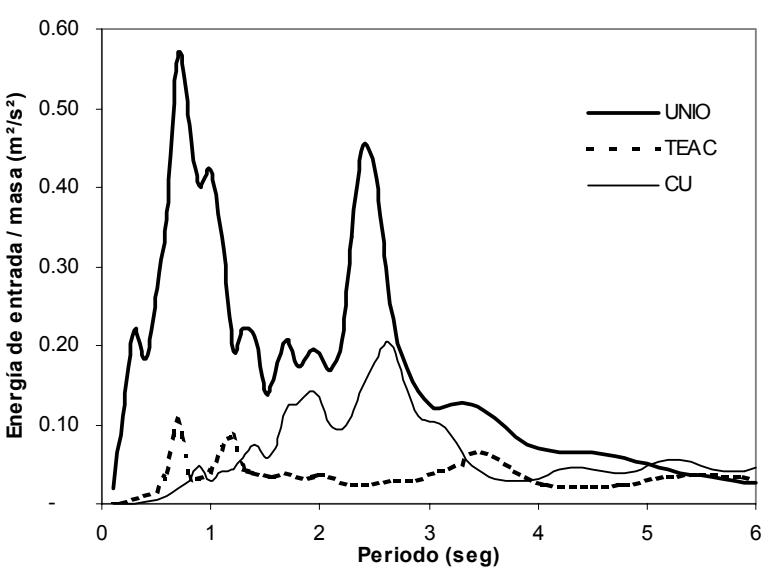

(d) UNIO, TEAC y CU (comp. N-S)

Figura 9. Espectros elásticos de energía de entrada para algunas estaciones que registraron el componente este-oeste del sismo del 19 de septiembre de $1985, \xi=5 \%$

Varios investigadores han observado que los sitios en terreno firme dentro del valle de México no siguen patrones de atenuación estándar, y que existe amplificación debida a efectos regionales (Ordaz y Singh, 1992; Reinoso, 1996; Reinoso y Ordaz, 1999). Esta amplificación también se observa en los espectros elásticos de energía de entrada $\left(E_{I}\right)$ mostrados en las figs. 9c y 9d para el sismo del 19 de septiembre de 1985 en el componente este-oeste y norte-sur, 
respectivamente. Las estaciones CALE y UNIO (fig 9a) se localizan sobre el área de ruptura y las estaciones TEAC y CU se localizan a 220 y $280 \mathrm{~km}$ de distancia de la superficie de ruptura, respectivamente. La estación TEAC se localiza fuera del valle de México y CU dentro de éste (fig. 10). Se observa que las ordenadas espectrales de energía en CU sufren amplificación sobre todo en periodos de 1 a 3 segundos, obteniéndose en algunos casos ordenadas de energía similares a las observadas en las estaciones del área epicentral (fig. 9c). Este efecto de amplificación aún en sitios de terreno firme del valle de México contribuye a que en este lugar la energía sísmica sea tan grande, como se verá a continuación.

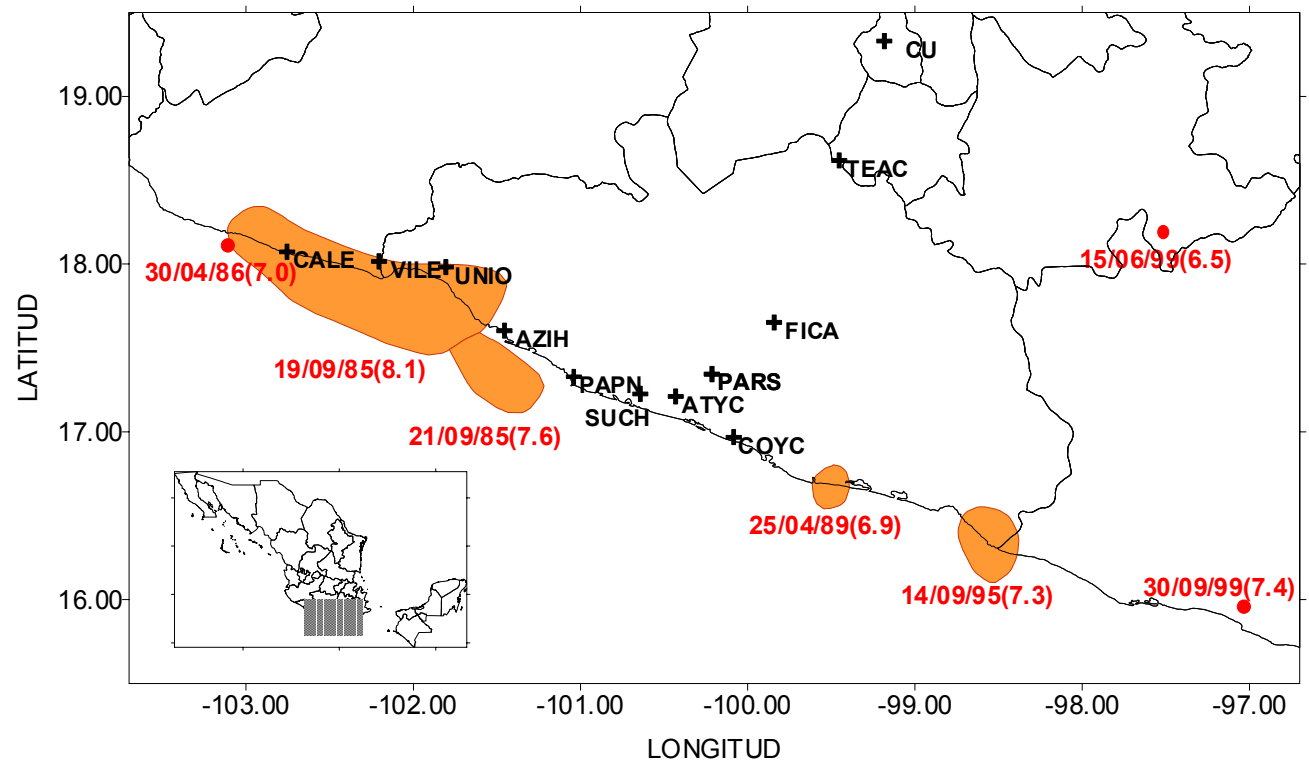

Figura 10. Localización de estaciones acelerométricas, de epicentros y de áreas de ruptura de sismos recientes

\section{ESPECTROS DE ENERGÍA EN EL VALLE DE MEXICO}

En el valle de México se manifiestan de forma dramática los efectos de amplificación dinámica en depósitos lacustres. La ciudad de México es particularmente vulnerable a sismos de subducción ocurridos en las costas del Pacífico. Las ondas sísmicas provenientes de esa región se atenúan en el trayecto hacia la ciudad de México, la cual se encuentra localizada a más de $250 \mathrm{~km}$ de distancia; sin embargo, al internarse a los estratos blandos originados por la desecación artificial de los antiguos lagos de Xochimilco, Texcoco, Xaltocan, Zumpango y Chalco, se presenta una importante amplificación de las ondas y un significativo incremento en la duración del movimiento dominada por el arribo de ondas de periodo largo que sufren menos atenuación que las de periodo corto.

A partir de 1985 la red acelerométrica de la ciudad de México creció considerablemente instrumentándose gran parte del valle. La fig. 11 muestra la zonificación geotécnica así como la ubicación de las estaciones, en donde se observa una mayor concentración de éstas en las zonas 
donde históricamente se han presentado mayores daños durante sismos intensos. En esta figura también se distinguen las principales avenidas de la ciudad.
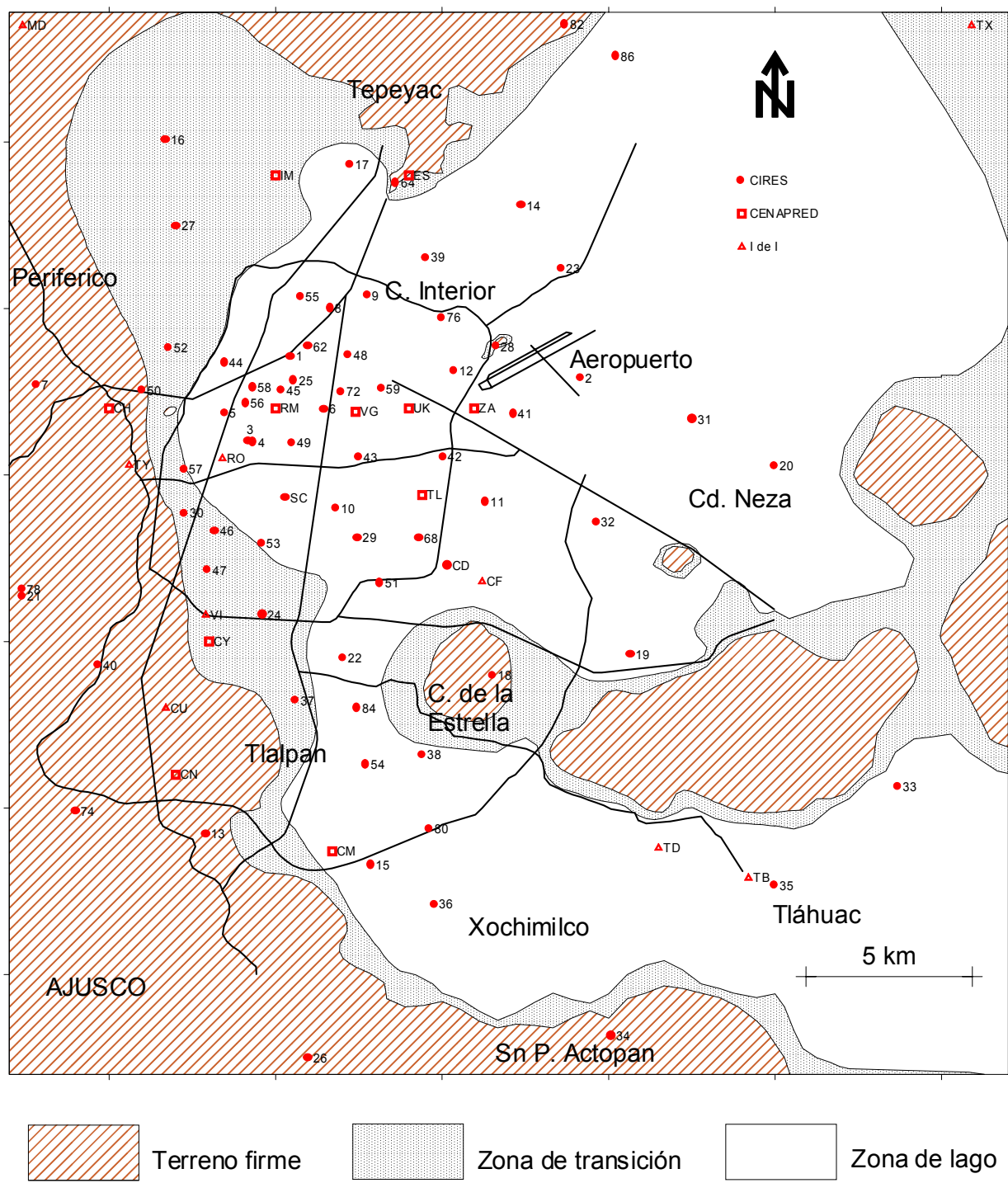

Figura 11. Zonificación geotécnica, algunos sitios de referencia y estaciones de la red acelerométrica de la ciudad de México

Contrario a lo que se mencionó en la primera parte de este trabajo al referirnos al movimiento sísmico en la zona epicentral, en cada estación acelerométrica del valle de México la mayoría de los parámetros sísmicos se correlacionan con el daño. Aunque existen grandes diferencias entre las estaciones debidas a los efectos de sitio, si analizamos cada estación por separado observamos que la aceleración del terreno y espectral, la velocidad, los desplazamientos, el contenido de frecuencias y la duración, para cada una de ellas, están directamente correlacionados con la magnitud (a mayor magnitud $>$ respuesta, y viceversa) e inversamente correlacionados con la distancia al área de ruptura (a mayor distancia $<$ respuesta, y viceversa). Es decir, el daño en cada sitio será mayor para magnitudes grandes y menores distancias. 
Los espectros de energía son especialmente sensibles a esta amplificación del movimiento. Para ilustrar estos efectos en la fig. 12 se muestran los espectros elásticos de energía de entrada $\left(E_{I}\right)$ del sismo del 19 de septiembre de 1985 componente este-oeste para algunas estaciones localizadas en los diferentes tipos de suelo del valle de México. Las estaciones SCT y Central de Abastos (CD) se localizan en terreno blando, las estaciones de Viveros (VI) y CU se localizan en zona de transición y suelo firme, respectivamente. Se observa que los efectos de amplificación son bastante evidentes para las estaciones SCT y CD. Por ejemplo, los valores máximos de cada espectro son los siguientes: la estación SCT tiene $E_{\operatorname{lmax}}=22.7 \mathrm{~m}^{2} / \mathrm{s}^{2}$, la estación CD tiene $E_{\text {Imax }}=13.7 \mathrm{~m}^{2} / \mathrm{s}^{2}$ y en las estaciones VI y CU tienen un valor de aproximadamente 0.2 $\mathrm{m}^{2} / \mathrm{s}^{2}$. Se observa que los valores máximos en terreno blando son muy superiores a las zonas de terreno firme y transición ya que en las estaciones SCT y CD se registraron valores máximos de 113 y 68 veces el valor obtenido en las estaciones VI y CU.

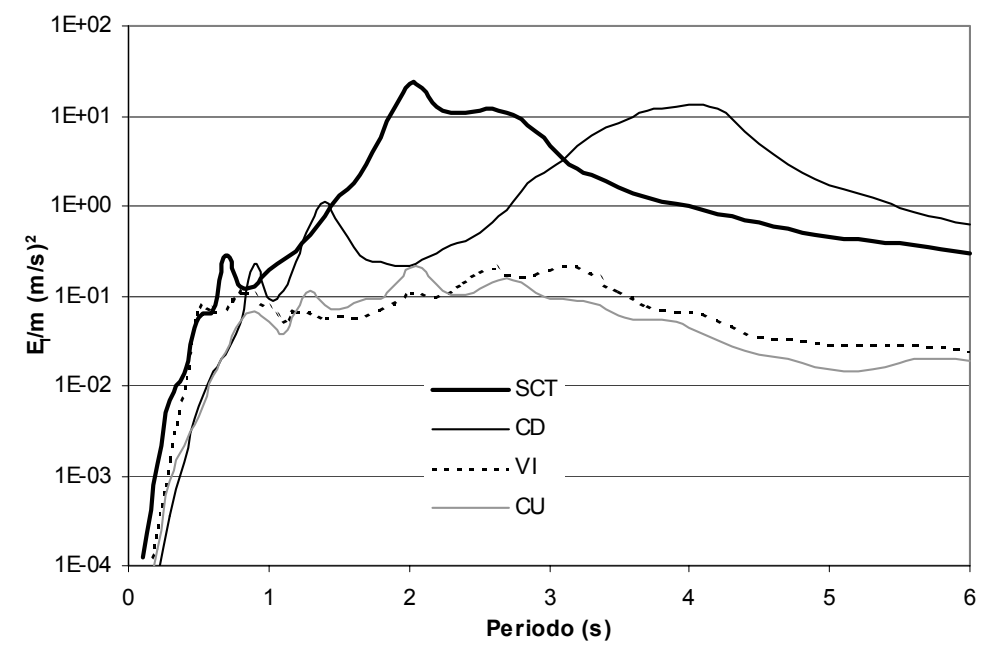

Figura 12. Espectros elásticos de energía de entrada en estaciones localizadas en diferentes tipos de suelo para el sismo del 19 de septiembre de $1985, \xi=5 \%$, en escala logarítmica

\section{Direcciones principales de energía}

La mayoría de los registros de aceleración de campo libre se obtienen convencionalmente en una dirección vertical y en dos direcciones horizontales perpendiculares entre sí: norte-sur y esteoeste. Es de esperarse que exista una dirección del movimiento en que la energía sea mayor y que no coincida con las que convencionalmente se escogieron. Esto se muestra en la fig. 13 para dos registros de la estación SCT, para el sismo del 19 de septiembre de 1985 y del 25 de abril de 1989. Por ejemplo para el 19 de septiembre (fig. 13a) la mayor ordenada de energía en la dirección principal corresponde a $29.7 \mathrm{~m}^{2} / \mathrm{s}^{2}$, la menor energía representa el $18 \%$ de este valor $\left(5.3 \mathrm{~m}^{2} / \mathrm{s}^{2}\right)$. Estos resultados podrían tener una repercusión importante en el diseño de estructuras si se logra caracterizar la dirección de la energía principal para distintos eventos sísmicos y de qué orden será el cociente entre la energía mayor y menor. Con ello se podrán utilizar reglas más eficientes sobre las fuerzas sísmicas en los dos componentes ortogonales de las estructuras. Por 
simplicidad, en este trabajo los cálculos de los espectros se realizaron tomando la dirección nortesur.

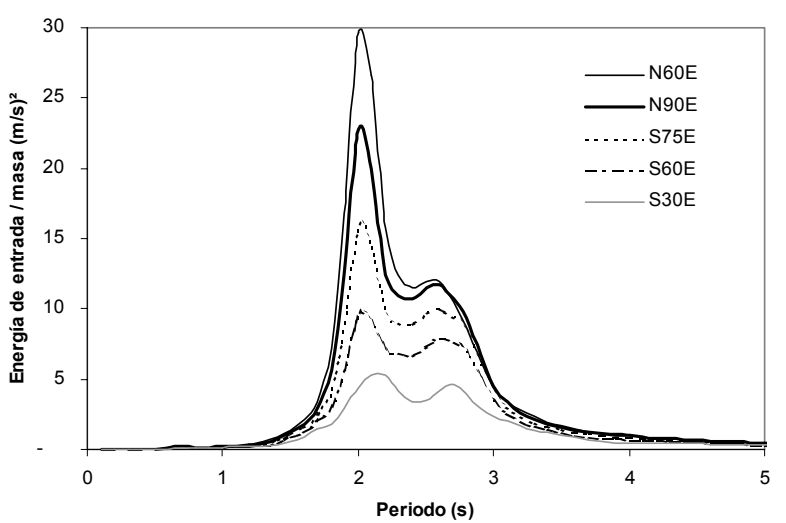

(a) 19 de septiembre de 1985

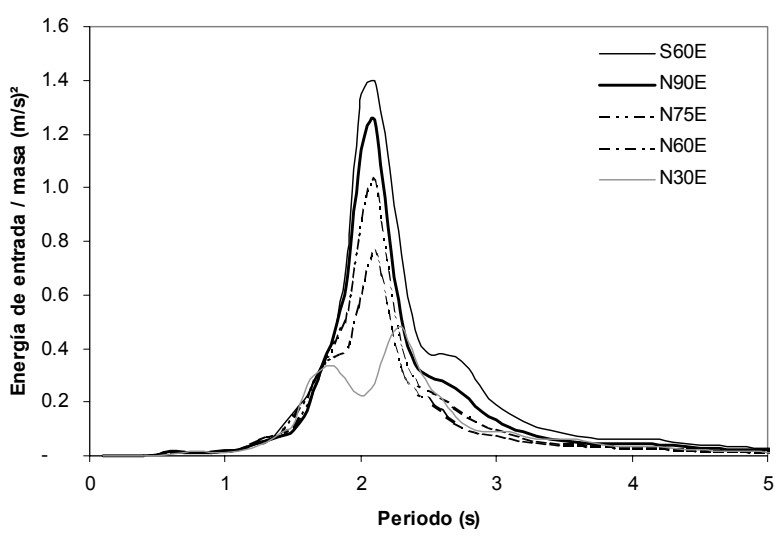

(b) 25 de abril de 1989

Figura 13. Variación de los espectros elásticos de energía de entrada con la dirección del movimiento, estación SCT, $\xi=5 \%$

\section{Influencia de la magnitud del sismo}

Con el propósito de observar la influencia de la magnitud del sismo en los espectros de energía en el valle de México, en la fig. 14 se muestran los espectros elásticos de energía de entrada en las estaciones SCT y Central de Abastos (CD) correspondientes a varios sismos de subducción. Los epicentros de estos sismos se muestran en el mapa de la fig. 10 con sus respectivas magnitudes. Se puede observar que casi todos las áreas de ruptura tienen una distancia a la ciudad de México similar $(\mathrm{R} \approx 300 \mathrm{~km})$ excepto por los epicentros de los sismos del 30 de abril de 1986 y del 30 de septiembre de 1999, cuya distancia es mayor. La fig. 14 muestra la diferencia sobresaliente de las ordenadas del espectro correspondiente al sismo del 19 de septiembre de $1985\left(22.7 \mathrm{~m}^{2} / \mathrm{s}^{2}\right.$ para SCT y $19.7 \mathrm{~m}^{2} / \mathrm{s}^{2}$ para CD) con respecto a los otros sismos. Esto explica en parte los daños ocasionados por este sismo en las estructuras localizadas en estas estaciones con periodos cercanos al pico del espectro correspondiente. Para los demás sismos cuyas magnitudes oscilan entre 6.9 y 7.6 , las ordenadas máximas son muy similares $\left(1.5\right.$ a $\left.2.5 \mathrm{~m}^{2} / \mathrm{s}^{2}\right)$ excepto para el sismo del 30 de abril de $1986\left(0.4 \mathrm{~m}^{2} / \mathrm{s}^{2}\right)$, lo que es atribuible a su mayor distancia a la ciudad de México. Se observa que los niveles de energía de las estaciones SCT y CD para los sismos de magnitudes menores o iguales a 7.6 grados están por debajo de $3.0 \mathrm{~m}^{2} / \mathrm{s}^{2}$. Se puede proponer dados los espectros y las observaciones de daño, que este nivel de energía no produce daño a las estructuras, aunque esta afirmación deba ser verificada mediante el uso de otros parámetros que se correlacionan mejor con el daño estructural como la energía histerética normalizada $\left(N E_{H}\right)$.

\section{Espectros inelásticos de energía}

Diseñar las estructuras para que su comportamiento sea elástico significa que no se presentarán daños estructurales ante los sismos considerados por el diseñador. Sin embargo, resulta 
sumamente costoso proporcionar los elementos de las dimensiones necesarias para lograr este comportamiento. En la práctica de diseño de estructuras no es común proyectar estructuras elásticas, por lo que es necesario balancear el daño permitido por comportamiento inelástico con el costo total de la estructura. Debido a esto surge la necesidad de calcular espectros que consideren las solicitaciones de la estructura correspondientes a comportamiento inelástico.

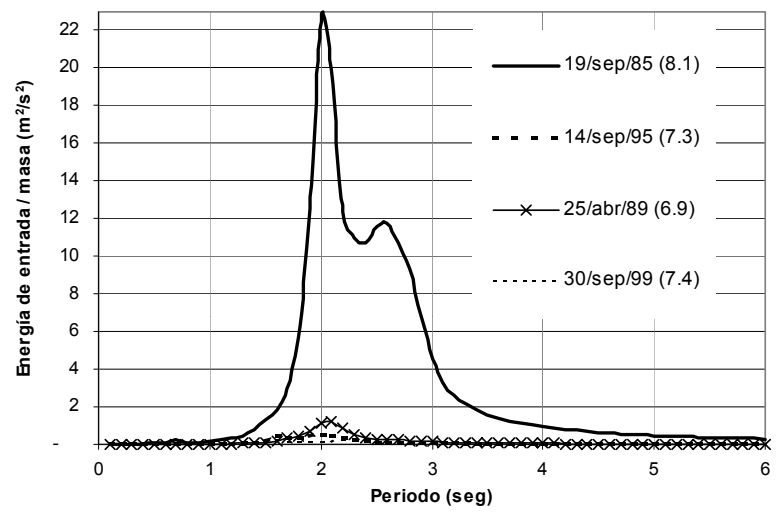

(a) Estación SCT

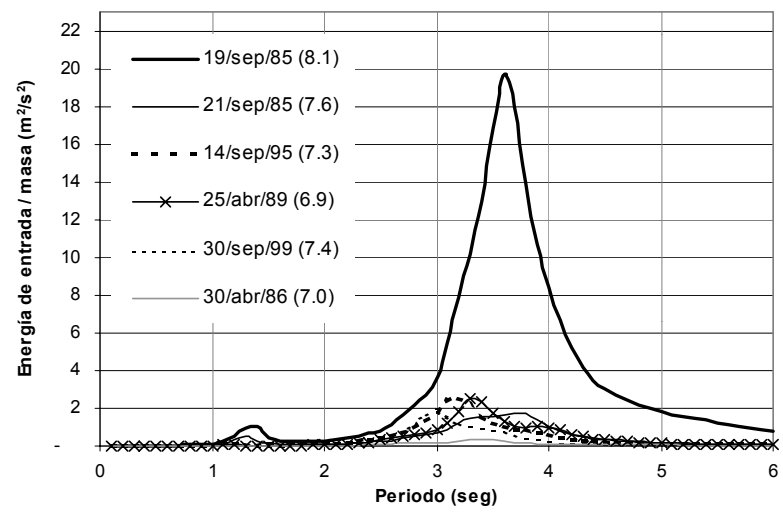

(b) Estación CD

Figura 14. Espectros elásticos de energía de entrada para sismos de varias magnitudes, $\xi=5 \%$

Los espectros inelásticos de energía más relevantes son los de entrada $\left(E_{I}\right)$ y los de histéresis $\left(E_{H}\right)$ ya que son los que se relacionan con la demanda de energía que impone el sismo a la estructura y con el daño que ésta puede sufrir. La fig. 15 muestra una representación espacial de estos espectros del registro de la estación SCT-EO durante el sismo del 19 de septiembre de 1985. En estos espectros se utilizó un eje adicional perpendicular al plano del espectro, el cual representa el coeficiente sísmico $C y$. Con ello se puede observar la variación de los espectros con diferentes resistencias. En estos cálculos se consideró un modelo de comportamiento elastoplástico perfecto y una fracción de amortiguamiento crítico $\xi=5 \%$.

La fig. 15a muestra la variación de los espectros de energía de entrada. Se observa que la traza de la superficie de esta gráfica en el plano vertical con $C y=1.2$ (mayor coeficiente sísmico) representa el espectro de energía de entrada con comportamiento elástico (fig. 14a). Conforme se reduce la resistencia del oscilador, $C y$, se incrementa la ductilidad y disminuye la energía de entrada debido a que la velocidad y la aceleración con que se mueve la masa del oscilador son menores por su comportamiento inelástico y es cuando aparece la energía de histéresis (fig. 15b). Los espectros inelásticos mostrados en la fig. 15 no tienen limitada la ductilidad, por lo que para valores muy bajos de $C y$ la ductilidad tiene valores grandes. Por ejemplo, para $C y=0.5$ la mayor demanda de ductilidad es de 1.33 , para $C y=0.2$ es de 2.78 y para $C y=0.1$ que es una resistencia común en edificios construidos en la ciudad de México ésta es aproximadamente de 20.0. Para periodos cortos existe una aparente ausencia de demanda de energía y en el periodo predominante del terreno se aprecia un cambio abrupto demandando gran cantidad de energía. Esto se debe a que en terreno blando la respuesta de estructuras con periodos cortos (la deformación relativa, velocidad y aceleración) son menores que para las estructuras de periodo mayores. Cuando el 
periodo de la estructura es muy cercano al del terreno la respuesta de la estructura sufre amplificación debido a que se presenta el fenómeno de resonancia.

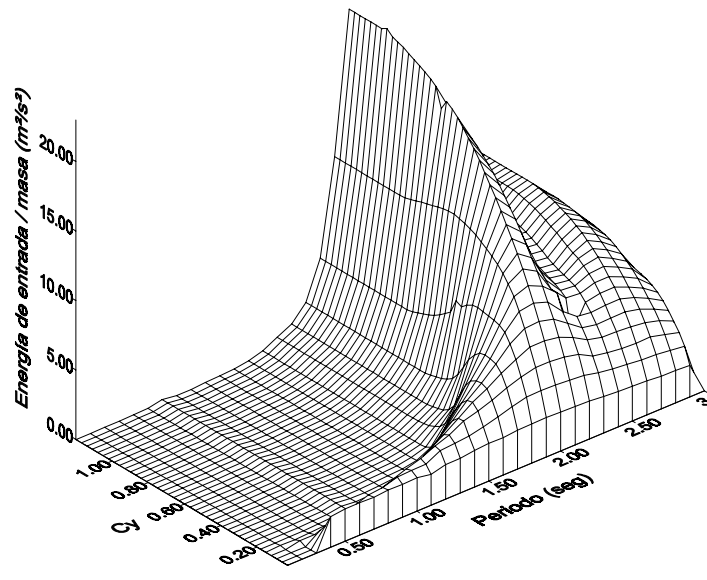

(a) Energía de entrada

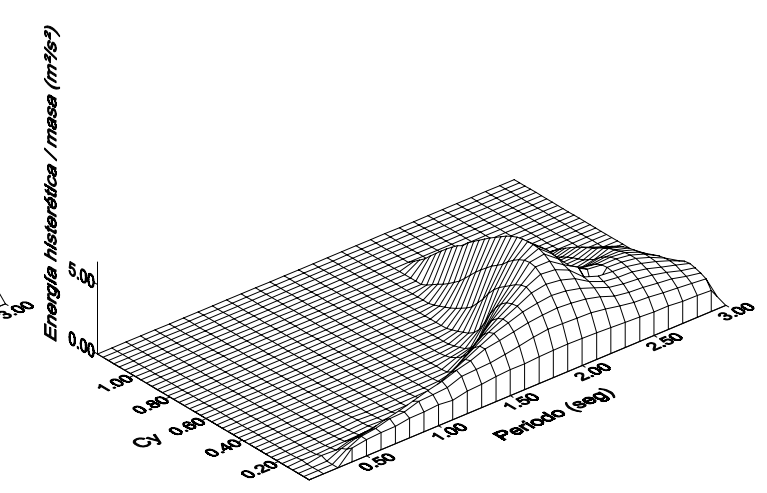

(b) Energía histerética

Figura 15. Representación espacial de la variación de los espectros de energía con la resistencia para el sismo del 19 de septiembre de 1985 en la estación SCT-EO, $\xi=5 \%$

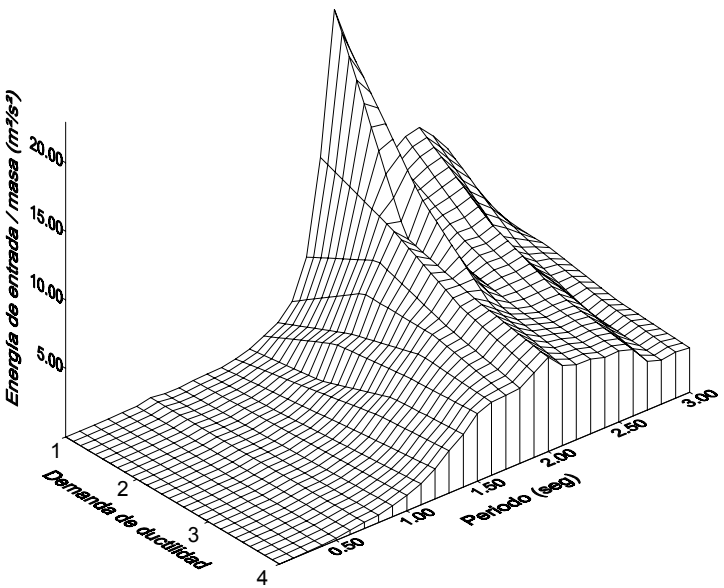

(a) Energía de entrada

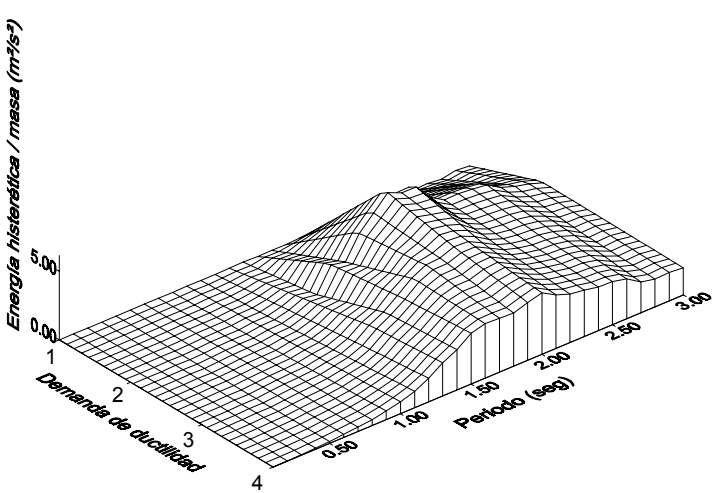

(b) Energía histerética

Figura 16. Representación espacial de la variación de los espectros de energía con demanda de ductilidad para el sismo del 19 de septiembre de 1985 en la estación SCT-EO, $\xi=5 \%$

En la fig. 16 se muestra una representación similar a la anterior, excepto que la variación de los espectros de energía es ahora con la demanda de ductilidad, $\mu$. En la fig. 16a se observa cómo existe una disminución de la energía de entrada en periodos cercanos al dominante del terreno y mayores a éste. Esta disminución es muy evidente entre los valores de $\mu=1$ a $\mu=2$ aproximadamente; sin embargo, para valores mayores a $\mu=2$ la energía es casi constante. Para 
algunos periodos menores al dominante se aprecia un ligero aumento de energía con una tendencia similar a la de periodos mayores a partir de $\mu=2$. Se observa que la energía de histéresis (fig. 16b) aparece y se incrementa a partir de la ductilidad $\mu=1$, y su comportamiento es casi constante para valores mayores de $\mu=2$. Se observa que en un rango de valores de demanda de ductilidad $(2<\mu<4)$ la relación entre la energía de histéresis y la de entrada es una cantidad muy estable que depende principalmente del amortiguamiento viscoso y en menor proporción de la ductilidad y del modelo de comportamiento histerético (Akiyama 1985; Kuwamura y Galambos 1989; Fajfar y Vidic 1994). Así, en este intervalo de valores al conocer la relación de $E_{H} / E_{I}$, el espectro $E_{H}$ puede ser fácilmente obtenido del espectro $E_{I}$ y viceversa.

En las figs. 15 y 16 se observa que los espectros de energía de entrada y de histéresis presentan un pico en el periodo predominante del terreno, aproximadamente dos segundos para esta estación. De estos diagramas resulta evidente qué estructuras son más vulnerables en esta zona para este tipo de temblor (estructuras con periodos alrededor de 2 segundos).

\section{Características de los espectros de energía}

En la fig. 17 se muestran los espectros elásticos de energía de entrada $\left(E_{I}\right)$ y los de energía histerética normalizada $\left(N E_{H}\right)$ para una demanda de ductilidad, $\mu=4$. Estos espectros corresponden al componente norte-sur de los registros localizados en las estaciones del valle de México para el sismo del 25 de abril de 1989. En este trabajo no se muestra el componente esteoeste pero la mayoría de los espectros del componente norte-sur es mayor que el componente este-oeste. Se escogió este sismo por ser el más intenso desde 1985 y porque fue ampliamente registrado por la red. El propósito de mostrar todos los registros no es el de identificar la energía en cada estación, sino mostrar algunas de las principales características del comportamiento global del valle. Para la energía elástica de entrada $\left(E_{I}\right)$ de la fig. 17a se observa lo siguiente:

- Los espectros de energía en terreno blando presentan un pico bien definido en el periodo dominante del terreno

- Existe una gran variación en la magnitud de la energía entre distintas estaciones, incluso para estaciones sobre suelos con el mismo periodo dominante

- La estación con la mayor ordenada espectral corresponde a la estación 49 (Centro Médico Siglo XXI) que presenta el pico en un periodo igual a 3 segundos. La estación SCT considerada a nivel mundial como la estación más representativa de amplificación dinámica en valles aluviales no es la de mayor demanda de energía dentro del valle (línea más gruesa en color negro). De hecho, el pico de la estación SCT tiene una ordenada espectral de aproximadamente un octavo de la ordenada espectral máxima de la estación 49

- Las estaciones con mayores ordenadas espectrales, entre 2 y 3.2 segundos, se localizan en zonas en las que durante sismos grandes se han reportado daños considerables. Sin embargo, hay zonas de $T s \approx 1.5$ segundos donde ha habido muchos daños durante sismos pasados y que presentan baja energía

- En periodos menores a 1.8 segundos la demanda de energía es muy pequeña, incluyendo las estaciones localizadas en terreno firme y algunas zonas donde ha habido daños 

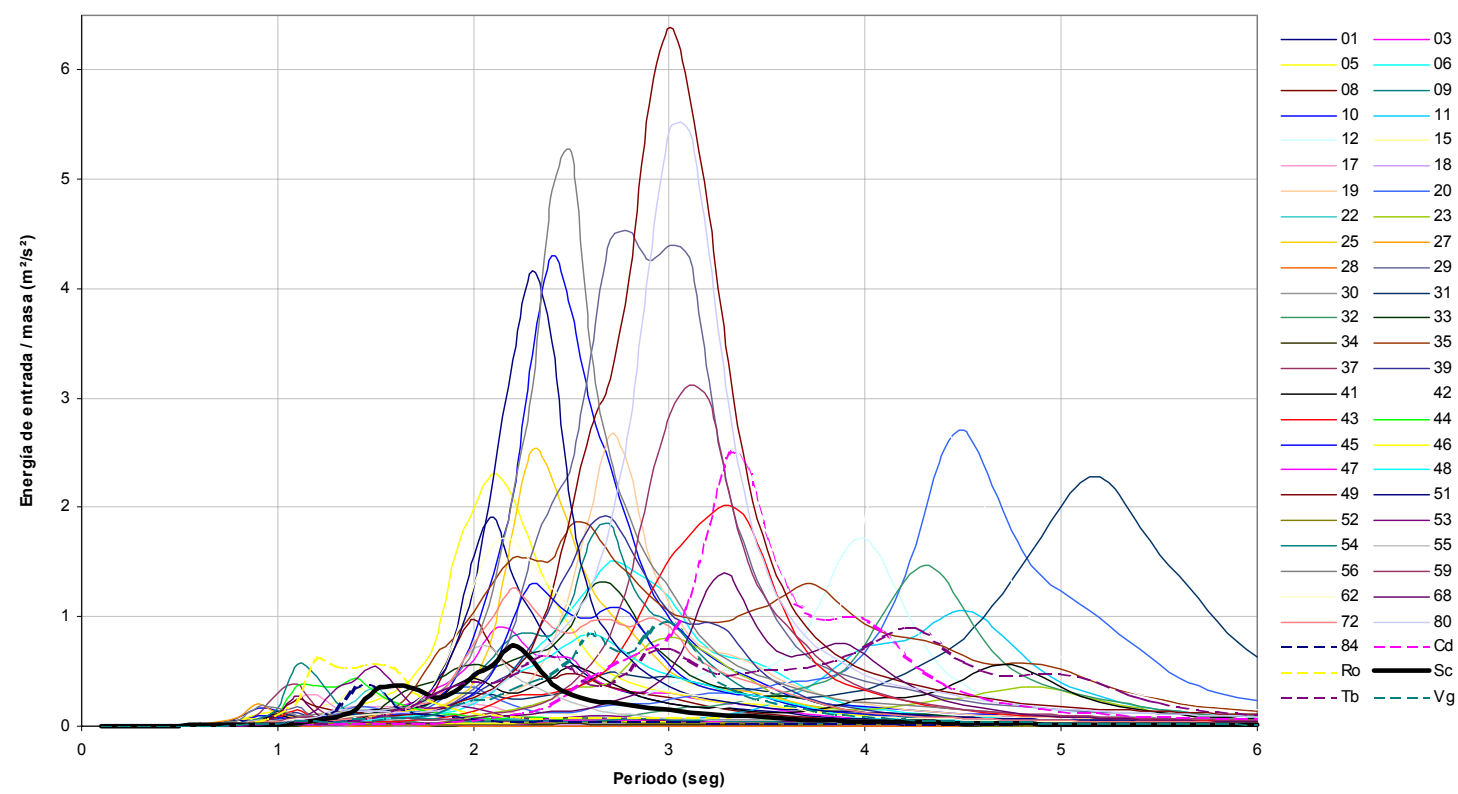

(a) Energía elástica de entrada

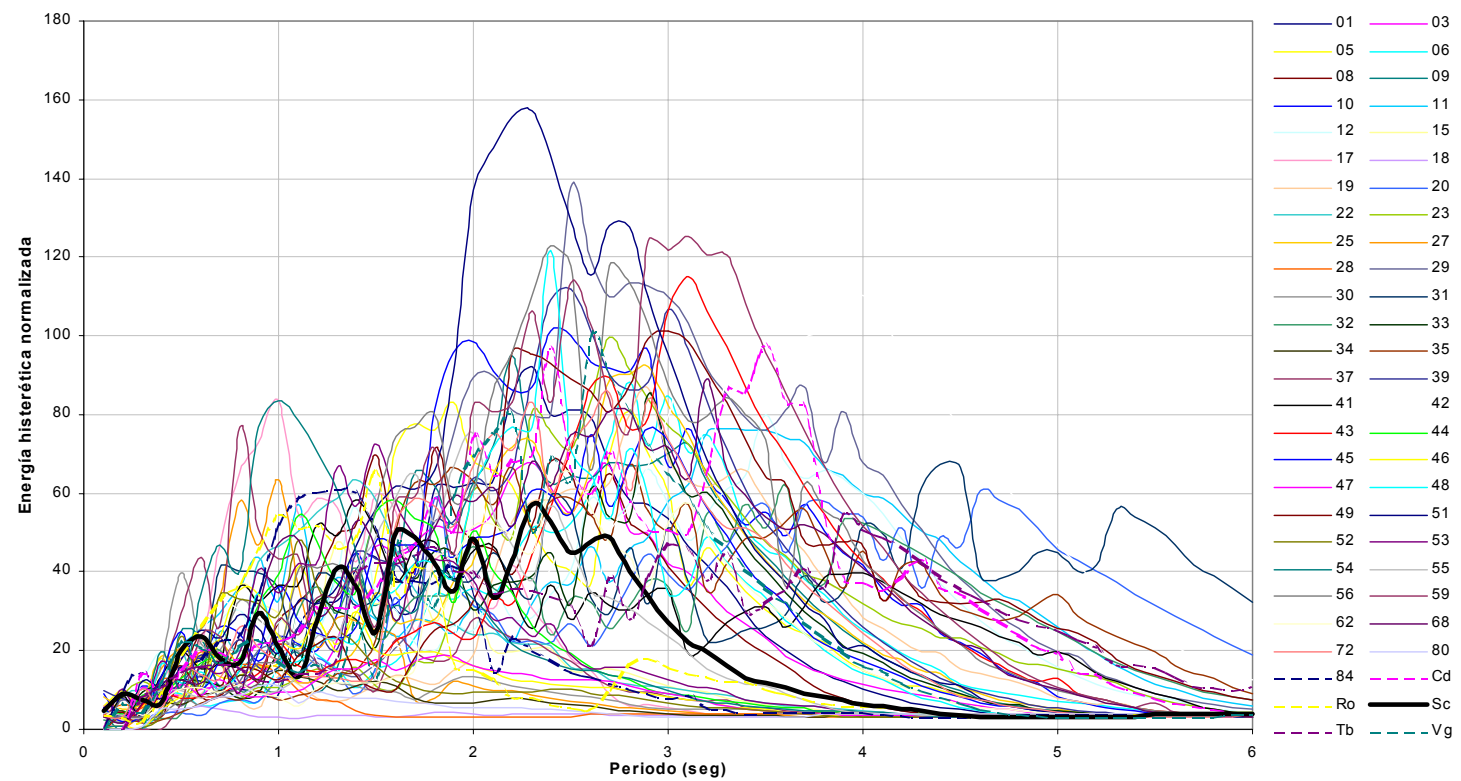

(b) Energía histerética normalizada, $\mu=4$

Figura 17. Espectros del sismo del 25 de abril de 1989 en la ciudad de México en el componente norte-sur, $\xi=5 \%$

Para la energía histerética normalizada $\left(N E_{H}\right)$ de la fig. $17 \mathrm{~b}$ se observa lo siguiente:

- Los espectros de energía histerética normalizada tienen forma irregular pero en muchos de los espectros la energía máxima se presenta en el periodo dominante del terreno 
- Las ordenadas de estos espectros no tienen tanta variación en amplitud como los espectros elásticos

- La estación con la mayor ordenada espectral corresponde a la estación 51, el pico se localiza en el periodo de 2.3 segundos. La estación SCT no es la que presenta mayores ordenadas pero tiene valores de ordenadas considerables (línea más gruesa en color negro)

- Los estaciones en que se presentan las mayores ordenadas espectrales son las mismas que las de los espectros elásticos (de periodos entre 2 y 3.2 segundos), pero ahora existen zonas de Ts $\approx 1.0$ segundos con valores de ordenadas importantes. Entre estas estaciones están algunas localizadas en zona de transición y en algunos casos tienen ordenadas superiores a las de terreno blando

Al analizar otros sismos (no se incluye en este trabajo) se tienen conclusiones muy similares, pero no siempre las mismas estaciones tienen las mayores ordenadas de energía.

\section{Curvas de igual energía en el valle de México}

Con los espectros mostrados en la fig. 17a calculados para el sismo del 25 de abril de 1989, se realizaron las curvas de igual energía elástica de entrada $\left(E_{I}\right)$ para varios intervalos de periodos estructurales. La fig. 18 muestra cuatro gráficas que corresponden a periodos estructurales de 2, 3,4 y 5 segundos, calculadas tomando el mayor valor en un intervalo de \pm 0.2 segundos en cada periodo. Las curvas corresponden a cuatro diferentes niveles de energía, del tono más claro al más oscuro, y corresponden a intervalos de 0.0 a 0.4 , de 0.4 a 1.0 , de 1.0 a 1.6 y mayor que 1.6 $\mathrm{m}^{2} / \mathrm{s}^{2}$, respectivamente. También se muestran con líneas oscuras las zonas de daño de los sismos de septiembre de 1985, las zonas rectangulares representan los límites de las zonas que presentaron daños y las otras zonas marcadas con línea oscura dentro de las áreas rectangulares representan las zonas de mayor daño. No se incluyen las zonas de daño de los sismos de 1957 y 1979. Sin embargo, estas zonas son muy similares a las observadas en la parte central de la ciudad de México durante los sismos de 1985.

Los niveles de energía usados son sólo para observar la variación de la energía y su distribución en el valle de México. Las gráficas más interesantes son las correspondientes a los periodos de 2 y 3 segundos, ya que son las que se correlacionan con zonas de peligro para estructuras comunes. No se incluyen las gráficas para periodos de un segundo ya que tienen ordenadas espectrales muy pequeñas. Para este sismo existe más energía de entrada en la parte oeste de la zona de lago que corresponde a los sitios que históricamente han mostrado ser los más afectados durante sismos de subducción a excepción de la zona con periodo del terreno Ts $\approx 1.5$ segundos localizada entre Tlalpan y Cerro de la Estrella. Se observa que al sur del valle de México (Xochimilco y Tláhuac) existen zonas con bastante energía $E_{I}$, donde deben tomarse medidas para evitar daños en sismos futuros. Conforme se incrementa el periodo estructural la energía se mueve hacia las zonas de estratos de arcilla más profundos con Ts mayores, debido a que la energía de entrada máxima corresponde al periodo más largo (dominante) del terreno. Es claro que estas zonas de gran energía no se correlacionan con los daños durante sismos pasados debido a que no existen en esos sitios estructuras con periodos tan largos. 


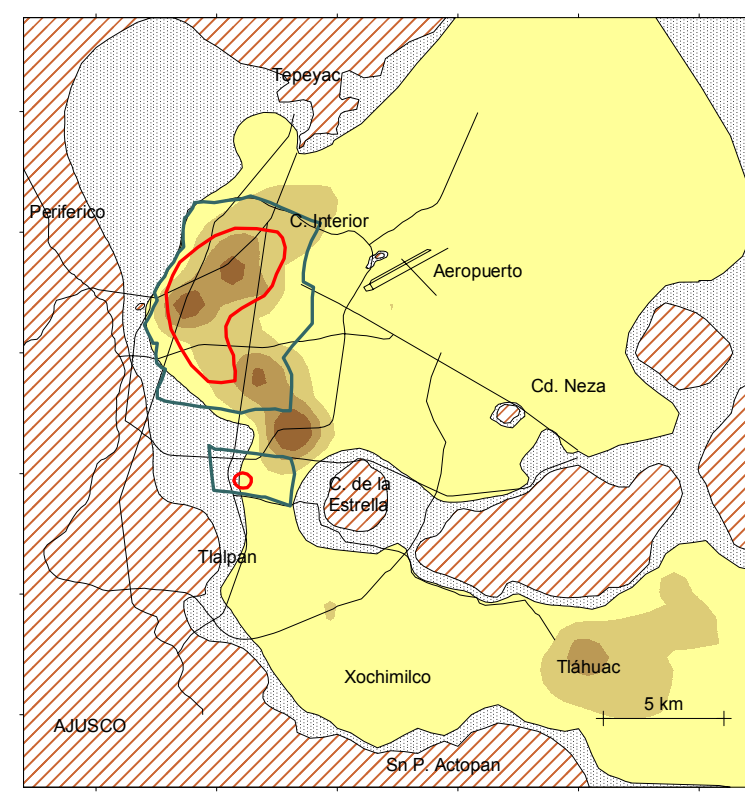

$\mathbf{T}=\mathbf{2} \operatorname{seg}$

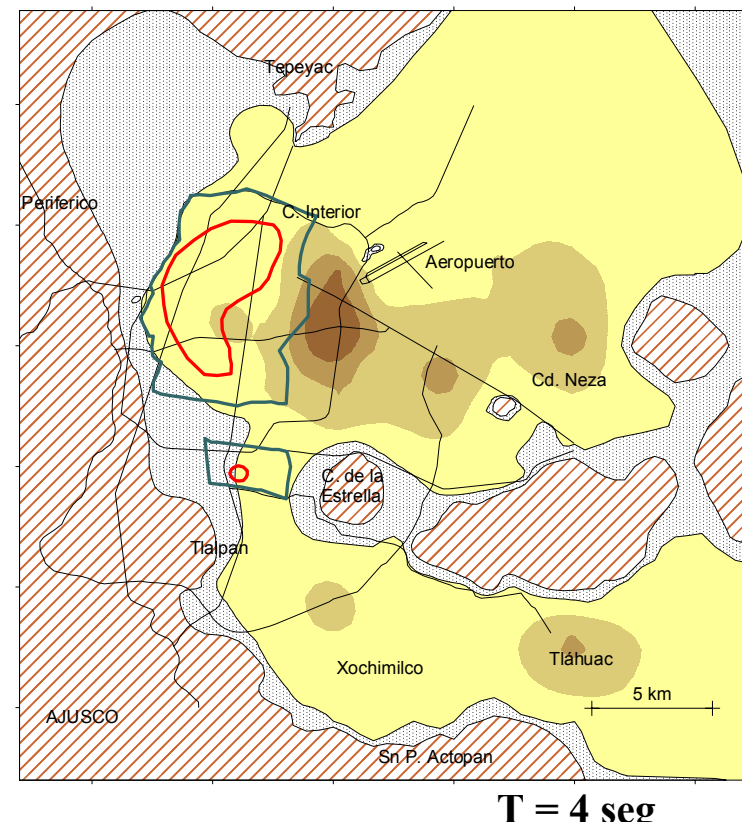

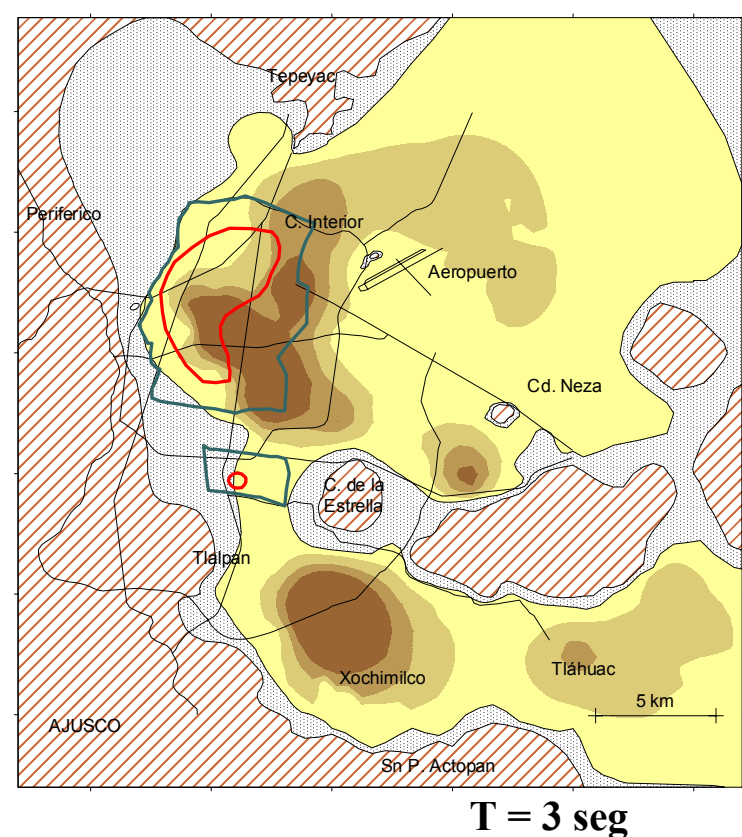

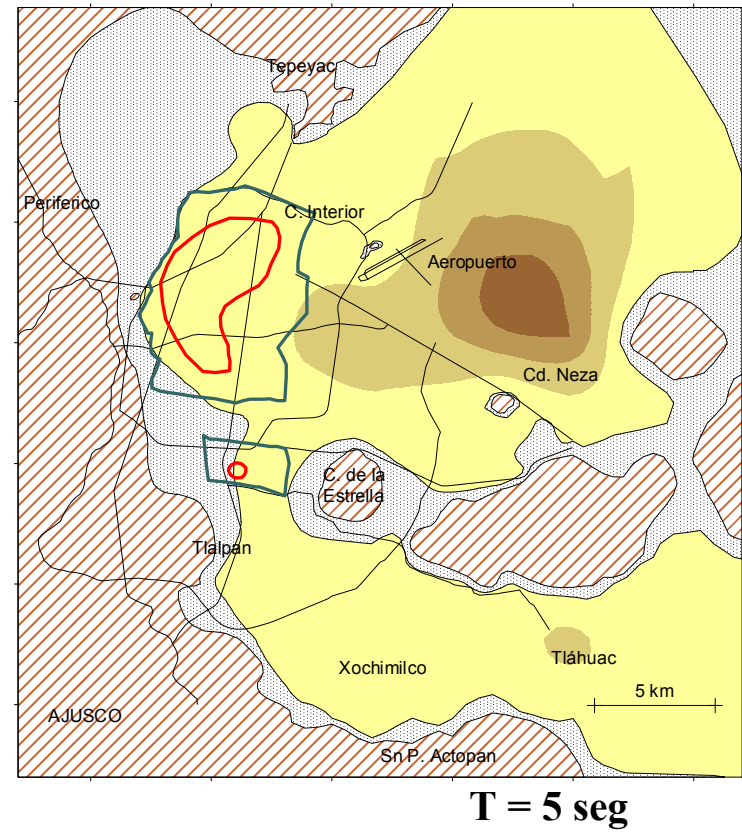

Figura 18. Curvas de igual energía elástica de entrada para cuatro periodos en el valle de México para el sismo del 25 de abril de 1989 y zonas de daño de los sismos de septiembre de 1985

Para los espectros mostrados en la fig. 17b también se realizaron las curvas de igual energía histerética normalizada $\left(N E_{H}\right)$ para varios intervalos de periodos estructurales. La fig. 19 muestra las gráficas que corresponden a periodos estructurales de 1, 2, 3 y 4 segundos, calculadas de la misma manera que la fig. 18. Estas curvas corresponden a intervalos de energía $N E_{H}$ de 0 a 35 , de 35 a 70 , de 70 a 105 y mayor que 105 , respectivamente. 

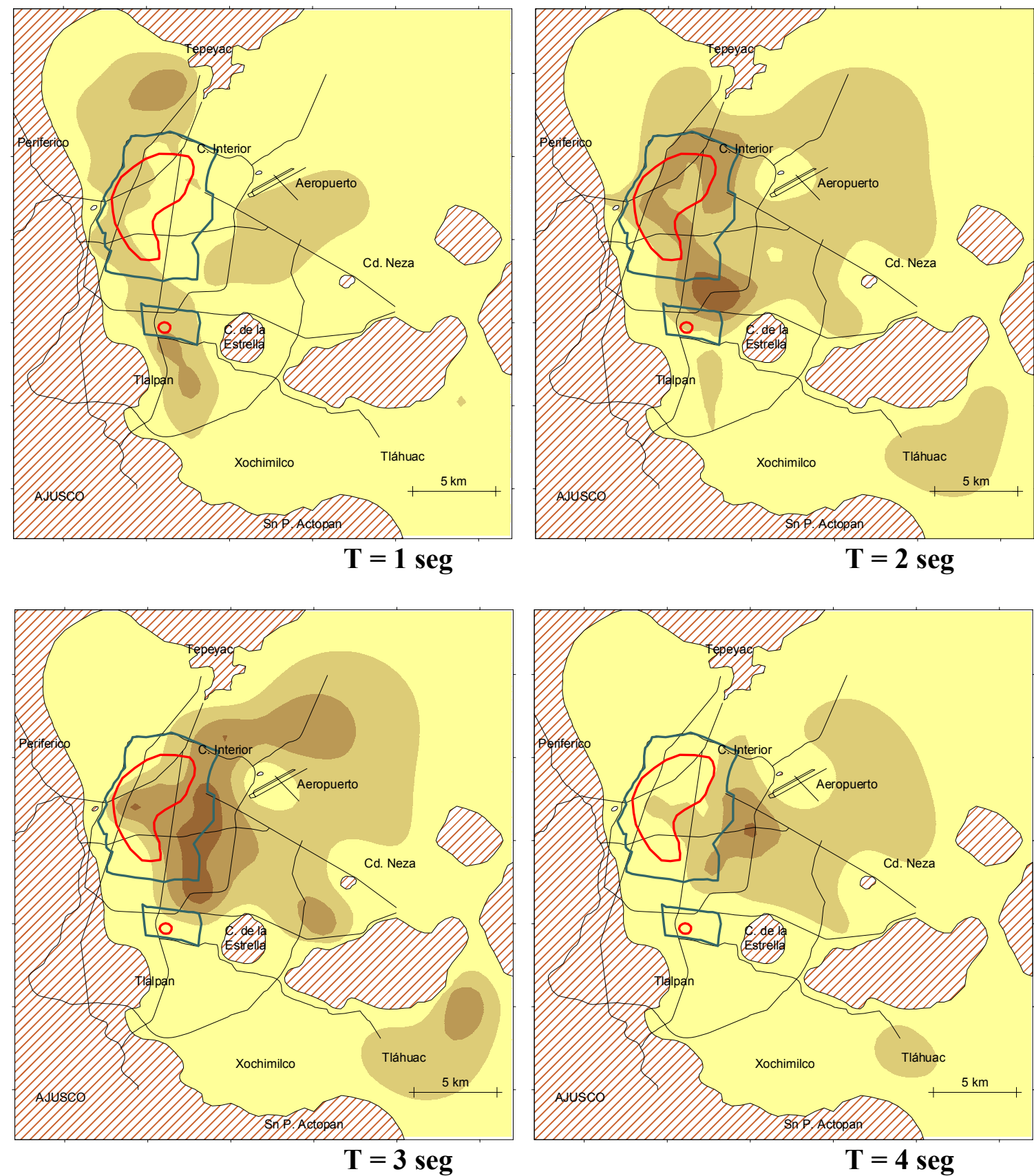

Figura 19. Curvas de igual energía histéretica normalizada $(\mu=4)$ para cuatro periodos en el valle de México para el sismo del 25 de abril de 1989 y zonas de daño de los sismos de septiembre de 1985

Es interesante hacer notar que para las gráficas correspondientes a periodo de un segundo se observan zonas que tienen valores grandes de este parámetro incluso en zonas de transición. En estas gráficas se observa una distribución más uniforme de las curvas. Sin embargo, para los periodos de 2 y 3 segundos, que son los que se correlacionan mejor con el daño en las gráficas de la fig. 18 , los valores máximos de energía $N E_{H}$ no coinciden con las zonas que fueron afectadas y 
sólo algunos valores intermedios de energía coinciden con las zonas de daños. A pesar de que existe energía $N E_{H}$ en la zona localizada entre Tlalpan y Cerro de la Estrella para periodos de 1 y 2 segundos, ésta tampoco tiene coincidencia con las zonas de daños que se registraron durante éste sismo. Esto se explica debido a que en la fig. 19 solo se muestran los mapas para cuatro periodos arbitrarios, un valor intermedio (como $\mathrm{T}=1.5 \mathrm{~s}$ para esa zona) mostrarían valores máximos en otros sitios. Al comparar las gráficas de $N E_{H}$ de periodo de 2 y 3 segundos con las correspondientes de $E_{I}$ se observa que las gráficas de energía elástica de entrada (fig. 18) tienen mayor correlación con el daño ocurrido en la parte norte del valle de México durante el sismo de 1985, siendo la gráfica de 2 segundos la que tiene más coincidencia con las zonas de daños de éste sismo, ya que no había, ni hay, estructuras con periodo largo en las demás zonas de gran energía (gráficas de $\mathrm{T}=3,4$ y 5 segundos).

En la fig. 20 se muestran las curvas de igual energía elástica de entrada $\left(E_{I}\right)$ en el valle de México realizadas de forma similar a las anteriores, pero normalizando la energía de todas las estaciones por la energía máxima que se registró en cada sismo. Estas curvas corresponden a cuatro sismos para un periodo estructural de 2 segundos ( 25 de abril de 1989, 14 de septiembre de 1995, 30 de septiembre de 1999 y del 15 de junio de 1999). En estas curvas los cuatro diferentes niveles de energía corresponden a intervalos de porcentaje de energía máxima del sismo de $0 \%$ a $10 \%$, de $10 \%$ a $20 \%$, de $20 \%$ a $40 \%$ y mayor que $40 \%$, respectivamente. Al igual que en las gráficas anteriores el nivel de energía sólo es para comparar las curvas de los diferentes sismos. Se observa que las zonas en que se presenta la energía máxima son recurrentes, con poca variación de un sismo a otro que puede ser atribuida a la magnitud, distancia al área epicentral y en menor escala a las características del sismo. De comprobar este patrón para sismos futuros, el promedio de estas curvas puede contarse como una herramienta más para la microzonificación de la ciudad.

\section{CONCLUSIONES}

En este trabajo se realizó una análisis de la variación de la demanda de energía en sitios cercanos a la zona epicentral y más detalladamente en el valle de México. Para la zona epicentral se confirmó el hecho de que los espectros de aceleración de los registros analizados incluyendo la aceleración de terreno no tienen una buena correlación ni con la magnitud del sismo ni con el daño que pueden sufrir las estructuras. La velocidad espectral está más correlacionada con el daño pero, el daño observado en las estructuras durante sismos recientes, incluyendo las de periodo corto, no puede atribuirse a estas pequeñas diferencias, por lo que se comprobó que utilizar la velocidad al cuadrado es un mejor parámetro de daño. Se mostró que existe una relación más estrecha de la magnitud del sismo con las ordenadas espectrales de energía de entrada.

En el valle de México existe una gran variación en la magnitud de la energía de entrada entre distintas estaciones, incluso para las estaciones que tienen suelos con el mismo periodo dominante. En algunas de ellas la energía presenta gran variación con la dirección del movimiento. Las estaciones con mayores ordenadas espectrales se localizan en zonas donde en sismos grandes se han reportado daños. 


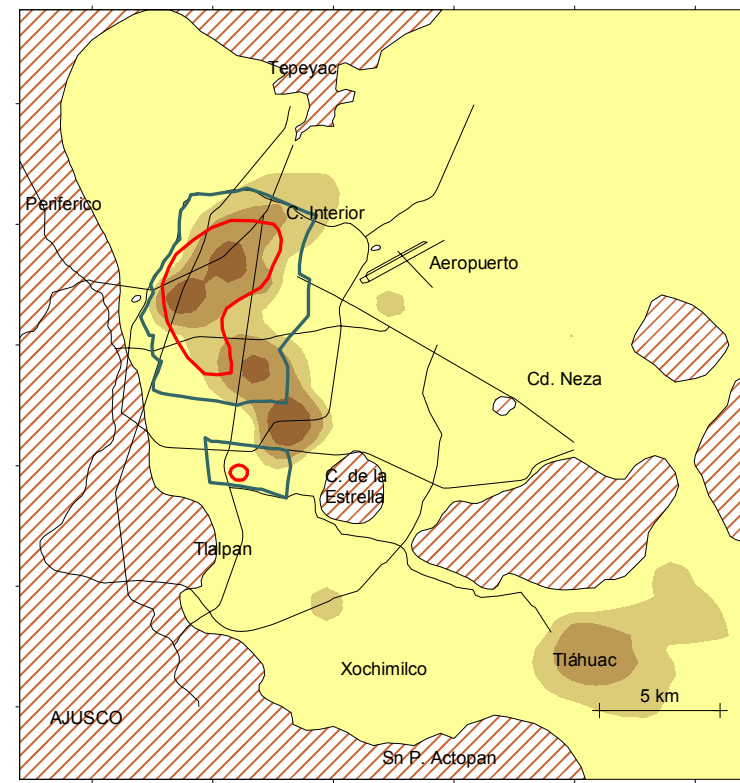

25 de abril de 1989

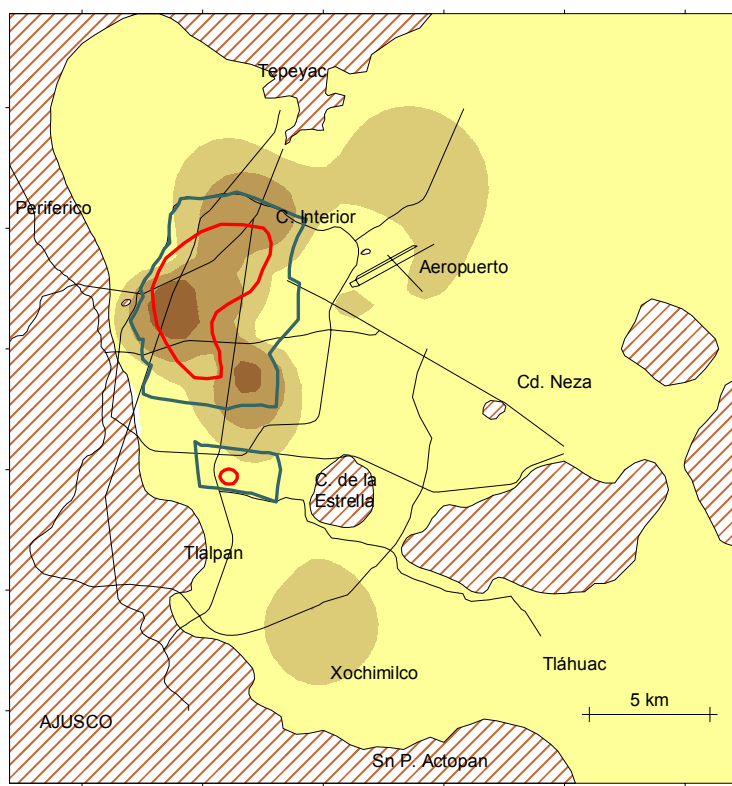

30 de septiembre de 1999

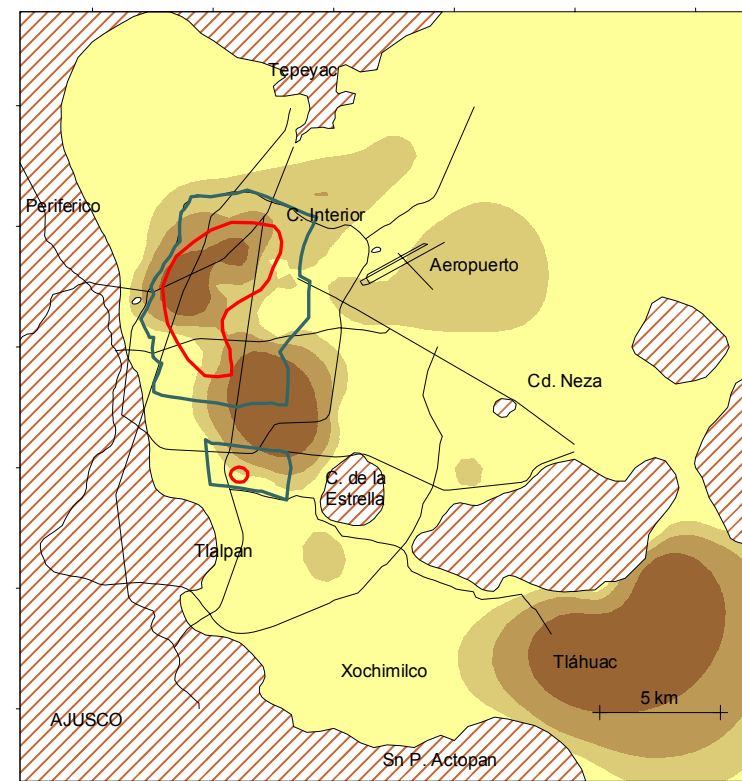

14 de septiembre de 1995

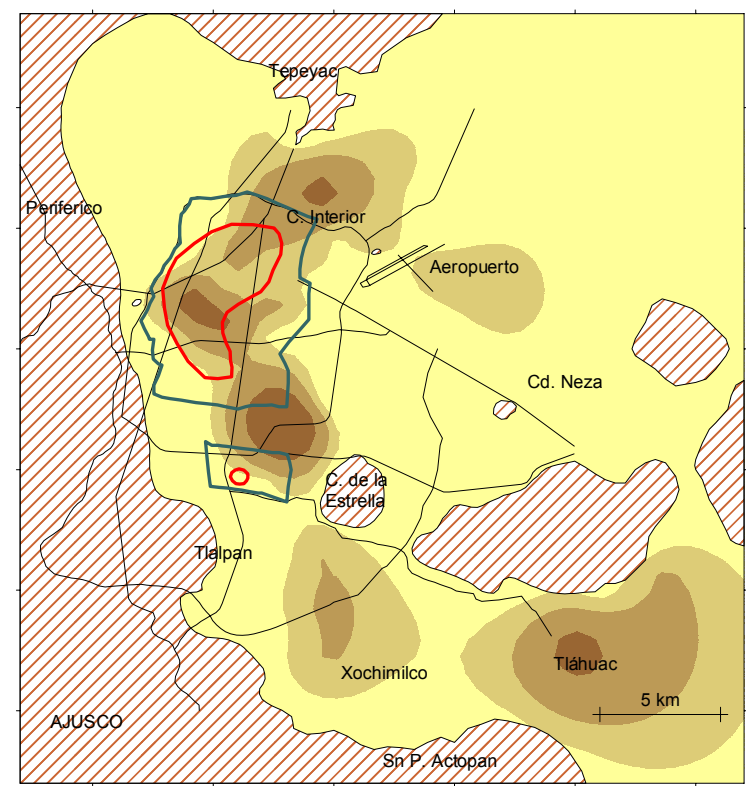

15 de junio de 1999

Figura 20. Curvas de igual energía de entrada en el valle de México para un periodo de dos segundos $(\mathrm{T}=2 \mathrm{~s})$ para cuatro sismos y zonas de daño de los sismos de septiembre de 1985

Para el sismo del 25 de abril de 1989 las estructuras con periodo de 3 segundos localizadas en la parte oeste de la zona de lago presenta mayores ordenadas espectrales de energía de entrada. Al comparar las gráficas de periodo de 2 y 3 segundos con las correspondientes de energía histerética normalizada se observa que existen algunas coincidencias de las zonas de mayor energía. Para este sismo existe más energía de entrada e histerética normalizada en sitios que históricamente han mostrado ser los más afectados durante sismos de subducción. Las 
gráficas de energía elástica de entrada tienen mayor correlación con el daño ocurrido en el valle de México durante el sismo de 19 de septiembre de 1985, siendo la gráfica de 2 segundos la que tiene más coincidencia con las zonas de daños de éste sismo. Las curvas obtenidas para cuatro diferentes sismos para un periodo de 2 segundos muestran que las zonas en que se presenta la energía máxima son recurrentes, y la poca variación puede ser atribuida a la magnitud, distancia al área epicentral, y en menor escala a las características del sismo.

La energía de entrada elástica es un parámetro que captura las características de la historia de amplitudes en el tiempo y duración del movimiento del terreno. Su uso en análisis probabilísticos de peligro sísmico puede proveer un mejoramiento en las bases de selección de escenarios sísmicos. Se considera que la energía elástica de entrada y la energía histerética normalizada pueden emplearse para mejorar la zonificación sísmica de la Ciudad de México y considerar aspectos de energía y duración del movimiento que hasta hoy no se ha tomado en cuenta.

\section{REFERENCIAS}

Akiyama, H (1985) "Earthquake Resistant Limit-State Design for Buildings", Universidad de Tokio Press. Japón.

Ávila, E y A Terán-Gilmore (2000) "Efecto de la Historia de Disipación de Energía en el Desempeño Estructural”, Memorias XII Congreso Nacional de Ingeniería Estructural, León Gto.

Bertero, V V (2000) "Performance-Based Seismic Engineering: Conventional vs. Innovative Approaches", Memorias 12vo Congreso Mundial de Ingeniería Sísmica, Nueva Zelanda, No. 2074.

Chou, C y C M Uang (2000) "Establishing absorbed energy spectra: an attenuation approach", Earthquake Engineering \& Structural Dynamics, 29, 10, pp 1441-1455, octubre.

Fajfar, P y H Krawinkler (1992) “On energy demand seismic in SDOF system”, Nonlinear Seismic Analysis and Design of Reinforced Concrete Buildings, Elsevier Applied Science, Nueva York.

Fajfar, P y T Vidic (1994) "Consistent Inelastic Design Spectra: Hysteretic and Input Energy", Earthquake Engineering \& Structural Dynamics, 23, 5, pp 523-537, mayo.

Housner, G W (1956) "Limit Design of Structures to Resist Earthquakes”, Memorias del Primer Congreso Mundial de Ingeniería Sísmica, Berkeley, California, pp 5-1 a 5-13.

Housner, G W (1975) "Measures of severity of earthquake ground shaking", Proceedings of the U.S. National Conference on Earthquake Engineering-1975, EERI, Oakland, California, pp 2533, junio.

Kuwamura, H y T V Galambos (1989) "Earthquake load for structural reliability", Journal of Structural Engineering, 115, 6, pp 1446-1462, junio.

Nau, J M y W J Hall (1984) "Scaling methods for earthquake response spectra", Journal of Structural Engineering, 110, 7, pp 1533-1548, julio. 
Ordaz, M. y S K Singh (1992) "Source Spectra and Spectral Attenuation of Seismic Waves from Mexican Earthquakes, and Evidence of Amplification in the Hill Zone of Mexico City", Bulletin of the Seismological Society of America, 82, 1, pp 24-43.

Popov, E P, T Yang y C Grigorian (1993) "New Directions in Structural Seismic Designs", Earthquake Spectra, Vol. 9, No. 4.

Reinoso, E (1996) "Algunos Resultados Recientes Sobre el Peligro Sísmico en la Ciudad de México", Revista de Ingeniería Sísmica, SMIS, No. 53, pp 1-24.

Reinoso, E y M Ordaz (1999) "Spectral Ratios for Mexico City from Free-Field Recordings", Earthquake Spectra, 15, 2, pp 273-295, mayo.

Reinoso, E y M Ordaz (2001) "Duration of Strong Ground Motion During Mexican Earthquakes in Terms of Magnitude, Distance to the Rupture Area and Dominant Site Period", Earthquake Engineering \& Structural Dynamics, 30: 653-673.

Terán-Gilmore, A (1996) "Performance-Based Earthquake-Resistant Design of Framed Buildings Using Energy Concepts", Tesis (Ph. Dr. en Ingeniería Civil) Universidad de California, Berkeley.

Uang, C M y V V Bertero (1988) "Use of Energy as a Design Criterion in Earthquake Resistant Design”, Report No. UCB/EERC-88/18, Earthquake Engineering Research Center, Universidad de California, Berkeley.

Uang, C M y V V Bertero (1990) "Evaluation of Seismic Energy in Structures", Earthquake Engineering \& Structural Dynamics, 19, pp 77-90. 